Research Article

\title{
Elastic Web Buckling Stress and Ultimate Strength of H-Section Beams Dominated by Web Buckling
}

\author{
Jiaxing Ma, ${ }^{1}$ Tao Wang $\mathbb{D}^{1}{ }^{1}$ Yinhui Wang, ${ }^{1}$ and Kikuo Ikarashi ${ }^{2}$ \\ ${ }^{1}$ School of Civil Engineering and Architecture, NingboTech University, Ningbo 315100, China \\ ${ }^{2}$ Department of Architecture and Building Engineering, Tokyo Institute of Technology, Tokyo 1528550, Japan \\ Correspondence should be addressed to Tao Wang; wangtao@nit.net.cn
}

Received 11 June 2020; Accepted 3 August 2020; Published 19 August 2020

Academic Editor: Francesco Cannizzaro

Copyright (c) 2020 Jiaxing Ma et al. This is an open access article distributed under the Creative Commons Attribution License, which permits unrestricted use, distribution, and reproduction in any medium, provided the original work is properly cited.

Numerical analyses and theoretic analyses are presented to study the elastic buckling of $\mathrm{H}$-section beam web under combined bending and shear force. Results show that the buckling stress of a single web with clamped edges gives a good agreement with the buckling stress of an $\mathrm{H}$-section beam web when the local buckling of the beam is dominated by the web buckling. Based on theoretic analyses, a parametric study is conducted to simplify the calculation of buckling coefficients. The parameters involved are clarified first, and the improved equations for the buckling coefficient and buckling stress are suggested. By applying the proposed method, the web buckling slenderness ratio is defined. It is verified that the web buckling slenderness ratio has a strong correlation with the normalized ultimate strength of $\mathrm{H}$-section beams when the buckling of the beams is dominated by web buckling. Finally, a design equation is proposed for the ultimate strength of $\mathrm{H}$-section beams.

\section{Introduction}

The main girder in a steel frame bears large bending moment and shear force due to the horizontal load and the secondary beam load as shown in Figure 1; the global buckling and local buckling may occur at the end of the main girder. Current stability design methods of the $\mathrm{H}$-section girder are mainly based on the calculation of elastic buckling stress. The elastic global buckling stress mainly depends on the interval of lateral supports and the width of flange, while the elastic local buckling stress mainly depends on the width-thickness ratio and depth-thickness ratio. However, the load condition and boundary condition may have a large influence on the buckling stress as well.

The theoretic studies on elastic local buckling have a long history: the traditional approach is to study the elastic buckling of a rectangular flat plate under assumed stress conditions and with various boundary conditions by using the energy method [1-5]. Yuan and Jin [6] proposed an extended Knatorovich method to solve the buckling problem of flat plates with various boundary conditions under compression and pure shear force and derived high-accuracy buckling coefficients. Kang and Leissa [7] formulated an exact solution procedure for the buckling analysis of flat plates with various boundary conditions under combined compression and bending force. Jana and Bhaskar [8] carried out buckling analyses of flat plates under nonuniform uniaxial compression by using Galerkin's method. To analyze the buckling stress of a web plate under complex tress conditions, Ritz's energy method using Fourier series functions is commonly adopted. Ikarashi and Suzuki [9] conducted Ritz's energy method to analyze the buckling stress of web plates with simply supported and clamped edges under combined bending and shear force, discovering that the results rapidly converged to the true solution as the number of Fourier series terms increased. Liu and Pavlovic [10, 11] conducted Ritz's energy method to analyze the buckling stress of simply supported flat plates under patch compression and arbitrary loads, founding that the idealization of simple supports yielded sound agreements to many plates problems, when a plate was attached to other plates.

As above, efforts have been carried out on the elastic buckling of the rectangular flat plates. However, precise 


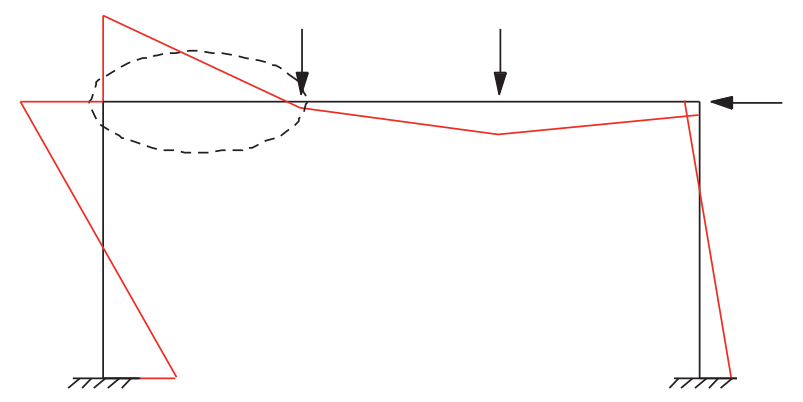

Figure 1: The moment diagram of a steel frame.

solutions for the buckling stress can only be obtained under simply assumed stress conditions, and it is still difficult to calculate the precise buckling stress of plates under complex stress condition, especially for the web of an $\mathrm{H}$-section beam under combined bending and shear force as shown in Figure 1. To calculate the buckling stress of plates under complex stress condition, the approximate method is often used. Based on the parametric study, Suzuki and Ikarashi [12] proposed a series of approximate equations to calculate the buckling stress of web under combined compression, bending, and shear force. The equations were found to be too complex to be applied for practical uses, due to the number of parameters involved. It is highly necessary to develop a design formula with high accuracies and simple calculations.

It is assumed that the local stability and the load bearing capacity of an $\mathrm{H}$-section beam are mainly dependent on the width-thickness ratio of the flange and depth-thickness ratio of the web. Kadono et al. [13] proposed the equivalent widththickness ratio which can be regarded as a major parameter to approximate the load bearing capacity of an $\mathrm{H}$-section beam. Kimura [14] proposed design equations for the ultimate strength and plastic deformation capacity by using the equivalent width-thickness ratio, which were useful when the buckling of the beam was dominated by flange buckling. However, this method has not been proved applicable for the web buckling dominant $\mathrm{H}$-section beams.

To evaluate the ultimate strength of web buckling dominant $\mathrm{H}$-section beams, the high-accuracy equation for the elastic buckling stress of web is required. This study aims to solve the eigenvalue problem for an $\mathrm{H}$-section beam web under combined bending and shear force by using Ritz's energy method and find out involved parameters. A parametric study is conducted to reveal the effect of parameters on the elastic buckling stress and to propose approximate equations for practical use. Based on the test results, the direct strength method is attempted to derive design equation for the ultimate strength of web buckling dominant $\mathrm{H}$-section beams by using the proposed equations of elastic buckling stress.

\section{Buckling Analysis}

2.1. Finite Element Analysis. The loading condition of the part of an $\mathrm{H}$-section beam (main girder) between the column and the secondary beam as shown in Figure 1 can be approximately regarded as Figure 2. The internal moment on the beam is assumed to be linearly varied along the length direction axis ( $x$-axis), which can be expressed as

$$
M(x)=\left(1-\frac{\beta}{L} x\right) M_{b},
$$

where $\mathrm{Mb}$ is the left end bending moment, $\beta$ is moment gradient $(0 \leq \beta \leq 2)$, and the right end bending moment is $(1-\beta) \mathrm{Mb}$. The relationship between $\mathrm{Mb}$ and shear load $Q_{s}$ is expressed as

$$
\beta M_{b}=Q_{s} L
$$

In this study, numerical simulations are conducted using the finite element program ABAQUS to analyze the $\mathrm{H}$-section beam. The boundary condition and load condition of an $\mathrm{H}$-section beam between the column and the secondary beam (Figure 1) are assumed as shown in Figure 3. The left beam end is connected with the column, and the right end with a stiffener is connected with the secondary beam. For simplification, a cantilever beam model using shell elements is used in FEA (Figure 3). The left end is completely fixed, the edges of web and flange on the right end are set as rigid edges to form a rigid plane, and the displacement along $z$-direction of the rigid plane is also constrained. On such boundary condition, the shear load $Q_{s}$ and moment $(1-\beta) \mathrm{Mb}$ are acting on the center point of the rigid plane in which $Q_{s}$ and $M_{b}$ satisfy equation (2). The FEA includes linear elastic buckling analysis and large deformation analysis. The elastic buckling analysis is performed first, and then the distribution of the initial geometric imperfection is assumed as the first elastic buckling mode obtained from the elastic buckling analysis.

The load condition of a beam end (Figure 1) is close to a cantilever beam with $\beta=1$. For this reason, numerous experimental studies on the $\mathrm{H}$-section cantilever beams have been reported [14-30], which have important reference significance for the study of local buckling. The shear span $L$ may be close to the interval of the secondary beam (Figure 1), and the normal value of $L / D$ should be approximately 4-6. According to the Code for Design of Steel Structure [31], for normal strength steel (Q235), $L / B$ should be no more than 16 to ensure the elastic global stability. In this paper, 158 sets of reported experimental data [14-30] of $\mathrm{H}$-section cantilever beams (with $\beta=1$ ) are collected as shown in Table 1. According to the data, it can be confirmed that, in most cases, $4 L / D \leq 6$ and $\mathrm{L} / \mathrm{B} \leq 16$. For comparison, two test results [30] and FEA results with normal dimensions are shown in Figure $4(L / D=1400 / 350=4, L / B=1400 /$ $175=8, t_{w}=4.5$, and $\left.t_{f}=16\right)$ and Figure $5(L / D=1400 /$ $350=4, L / B=1400 / 175=8, t_{w}=6$, and $\left.t_{f}=9\right)$. The load versus deflection response of the beam is generally affected by the initial geometric imperfection (IMP) and residual stress. As shown in Figures 4(a)-4(c) and Figures 5(a)-5(c), for both web buckling dominant and flange buckling dominant beams, the FEA results agree well with the test results, by employing proper initial geometric imperfections (with $\mathrm{IMP} \approx D / 400$ ). However, the actual distribution of the initial geometric imperfections and the residual stresses involved in the beams is unclear in the reported paper 


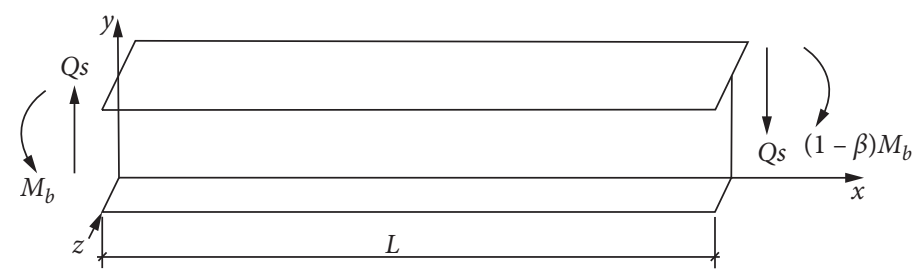

(a)

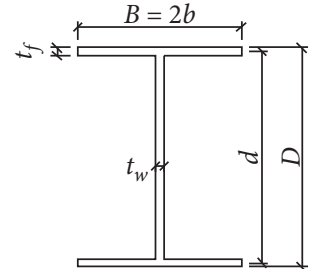

(b)

Figure 2: Load condition of $\mathrm{H}$-section beam.

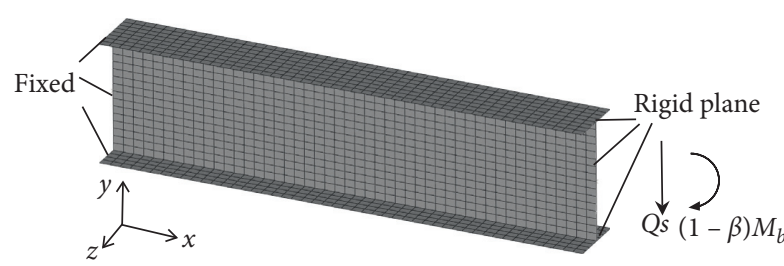

Figure 3: FEA model.

[14-30], and it is difficult to exactly analyze the load versus deflection response of the beams. In addition, the load versus deflection response is slightly affected by the loading program (including monotonic loading and various kinds of cyclic loading) as indicated by Kimura [14]. Thus, it is difficult to include these effects in the evaluation method to estimate the ultimate strength or the plastic deformation capacity of a beam. To estimate the ultimate strength of a beam, a simple calculation method is preferable for practical use.

Although the initial geometric imperfection, residual stress, loading program, and so on may cause a large deviation in the test, the relatively conservative evaluation method should be produced. In the following research, the direct strength method is conducted to investigate the correlation between the buckling slenderness ratio and the ultimate strength based on the test results [14-30] subjected to the web buckling dominant beam. The primary study is to propose high-accuracy formulas to calculate the elastic buckling stress.

2.2. Theoretic Analysis. To analyze the elastic buckling stress of a web, a simplified model conducted by the theoretical energy method is used here, in which the $\mathrm{H}$-section beam web is regarded as a single web with the boundary condition shown in Figure 6. The end edges $\mathrm{AB}$ and $\mathrm{CD}$ are set as rigid edges and constrained by a pin and a roller, respectively. The out-of-plane displacement of the other two longer edges AC and $\mathrm{BD}$ is constrained to keep straight, and the rotation around the $x$-axis is constrained as well. As shown in Figure 7 , the bending stress $\sigma(x, y)$ in the web can be expressed as

$$
\sigma(x, y)=\left(1-\frac{\beta}{L} x\right)\left(1-\frac{2}{d} y\right) \sigma_{b},
$$

where $\sigma \mathrm{b}$ is the maximum value of bending normal stress. $M_{b}$ can be expressed as

$$
M_{b}=\left[\frac{A_{w} d}{6}+A_{f}\left(d+t_{f}\right)\right] \sigma_{b},
$$

where $A_{w}=\mathrm{d} t_{w}$ is the section area of web and $A_{f}=2 b t_{f}$ is the section area of flange. The shear stress is assumed to be uniformly distributed, the shear force $Q_{s}$ is expressed as

$$
Q_{s}=A_{w} \tau_{s},
$$

where $\tau \mathrm{s}$ is the web shear stress. According to the above expression, the ratio of $\tau \mathrm{s}$ to $\sigma \mathrm{b}$ can be approximately expressed as

$$
\alpha=\frac{\tau_{s}}{\sigma_{b}}=\frac{\left((1 / 6)+\left(A_{f} / A_{w}\right)\right)}{\lambda_{w}},
$$

where $\lambda \mathrm{w}=L / d$ is the aspect ratio of web. The total potential energy of the web under combined bending and shear force is

$$
\Pi=U-V_{b}-V_{s},
$$

where $U$ is the strain energy and $V_{b}$ and $V_{s}$ are the external work due to bending and shear force, respectively. $U, V_{b}$, and $V_{s}$ can be expressed as follows:

$$
\begin{aligned}
U= & \frac{1}{2} D_{w} \int_{0}^{L} \int_{0}^{d}\left\{\left(\frac{\partial^{2} W}{\partial x^{2}}\right)^{2}+\left(\frac{\partial^{2} W}{\partial y^{2}}\right)^{2}+2 v \frac{\partial^{2} W}{\partial x^{2}} \frac{\partial^{2} W}{\partial y^{2}}\right. \\
& \left.+2(1-v)\left(\frac{\partial^{2} W}{\partial x \partial y}\right)^{2}\right\} \mathrm{d} x \mathrm{~d} y, \\
V_{b}= & \frac{1}{2} \sigma_{\mathrm{crw}} t_{w} \int_{0}^{L} \int_{0}^{d}\left(1-\frac{\beta}{L} x\right)\left(1-\frac{2}{d} y\right)\left(\frac{\partial W}{\partial x}\right)^{2} \mathrm{~d} x \mathrm{~d} y, \\
V_{s}= & \tau_{\mathrm{crw}} t_{w} \int_{0}^{L} \int_{0}^{d} \frac{\partial W}{\partial x} \frac{\partial W}{\partial y} \mathrm{~d} x \mathrm{~d} y,
\end{aligned}
$$

where $W$ is the out-of-plane displacement function and $D_{w}$ is the flexural rigidity: 
Table 1: Test data.

\begin{tabular}{|c|c|c|c|c|c|c|c|c|c|c|c|}
\hline Number & $\begin{array}{c}L \\
(\mathrm{~mm})\end{array}$ & $\begin{array}{c}D \\
(\mathrm{~mm})\end{array}$ & $\begin{array}{c}B \\
(\mathrm{~mm})\end{array}$ & $\begin{array}{c}t_{w} \\
(\mathrm{~mm})\end{array}$ & $\begin{array}{c}t_{f} \\
(\mathrm{~mm})\end{array}$ & $\begin{array}{c}s_{w y} \\
(\mathrm{MPa})\end{array}$ & $\begin{array}{c}s_{f y} \\
(\mathrm{MPa})\end{array}$ & $\left(b / t_{f}\right)_{\mathrm{eq}}$ & $S_{w}$ & $\begin{array}{r}\tau_{\max } \\
\text { (test) }\end{array}$ & $\begin{array}{l}\text { Load (M. stands for monotonic load; } \\
\text { C. stands for cyclic load) }\end{array}$ \\
\hline \multicolumn{12}{|c|}{ Reported by Fukuchi and Ogura [15] } \\
\hline 1 & 1000 & 223.4 & 269.6 & 5.8 & 8.8 & 297 & 270 & 0.594 & 0.305 & 1.08 & M. \\
\hline 2 & 1000 & 223.7 & 269.5 & 5.6 & 8.9 & 297 & 270 & 0.591 & 0.323 & 1.07 & C. \\
\hline 3 & 1000 & 223.3 & 269.6 & 5.7 & 8.9 & 297 & 270 & 0.589 & 0.314 & 1.08 & C. \\
\hline 4 & 1000 & 223.6 & 269.5 & 5.8 & 8.9 & 297 & 270 & 0.588 & 0.307 & 1.08 & C. \\
\hline 5 & 1000 & 223.4 & 270 & 5.8 & 8.8 & 297 & 270 & 0.595 & 0.306 & 1.07 & C. \\
\hline 6 & 1000 & 223.4 & 288.1 & 8.8 & 12 & 322 & 262 & 0.451 & 0.190 & 1.24 & M. \\
\hline 7 & 1000 & 224.2 & 287.8 & 8.8 & 12 & 322 & 262 & 0.451 & 0.191 & 1.22 & C. \\
\hline 8 & 1000 & 223.9 & 287.8 & 8.9 & 12.1 & 322 & 262 & 0.447 & 0.188 & 1.19 & C. \\
\hline 9 & 1000 & 224.3 & 287.7 & 8.9 & 11.9 & 270 & 257 & 0.446 & 0.184 & 1.17 & C. \\
\hline 10 & 1000 & 224.5 & 287.8 & 8.9 & 11.9 & 270 & 257 & 0.447 & 0.184 & 1.21 & C. \\
\hline 11 & 1000 & 222.7 & 240.6 & 8.8 & 11.9 & 322 & 262 & 0.387 & 0.177 & 1.4 & M. \\
\hline 12 & 1000 & 222.7 & 240.6 & 8.8 & 11.9 & 322 & 262 & 0.387 & 0.177 & 1.26 & C. \\
\hline 13 & 1000 & 223.7 & 240.1 & 9.1 & 12.1 & 322 & 262 & 0.380 & 0.170 & 1.26 & C. \\
\hline 14 & 1000 & 225.1 & 239.6 & 8.9 & 12 & 270 & 257 & 0.376 & 0.173 & 1.29 & C. \\
\hline 15 & 1000 & 223.8 & 240 & 8.8 & 11.9 & 270 & 257 & 0.379 & 0.174 & 1.34 & C. \\
\hline 16 & 1000 & 222.7 & 192.7 & 8.9 & 12 & 322 & 262 & 0.318 & 0.162 & 1.47 & M. \\
\hline 17 & 1000 & 224.5 & 191.7 & 9.1 & 11.9 & 322 & 262 & 0.319 & 0.158 & 1.31 & C. \\
\hline 18 & 1000 & 223.8 & 190.8 & 8.8 & 11.7 & 322 & 262 & 0.324 & 0.164 & 1.34 & C. \\
\hline 19 & 1000 & 224 & 191.9 & 8.7 & 12 & 270 & 257 & 0.312 & 0.164 & 1.39 & C. \\
\hline 20 & 1000 & 225.2 & 192.1 & 8.9 & 11.9 & 270 & 257 & 0.313 & 0.160 & 1.43 & C. \\
\hline \multicolumn{12}{|c|}{ Reported by Kato et.al. [16] } \\
\hline 21 & 1040 & 198 & 144 & 6 & 9 & 291 & 291 & 0.349 & 0.211 & 1.36 & M. \\
\hline 22 & 1040 & 318 & 144 & 6 & 9 & 291 & 291 & 0.421 & 0.351 & 1.3 & M. \\
\hline 23 & 1040 & 438 & 144 & 6 & 9 & 291 & 291 & 0.510 & 0.490 & 1.19 & M. \\
\hline 24 & 1300 & 198 & 180 & 6 & 9 & 291 & 291 & 0.416 & 0.211 & 1.23 & M. \\
\hline 25 & 1300 & 318 & 180 & 6 & 9 & 291 & 291 & 0.478 & 0.351 & 1.22 & M. \\
\hline 26 & 2600 & 318 & 180 & 6 & 9 & 291 & 291 & 0.478 & 0.313 & 1.17 & M. \\
\hline 27 & 1300 & 438 & 180 & 6 & 9 & 291 & 291 & 0.558 & 0.491 & 1.1 & M. \\
\hline 28 & 1570 & 198 & 216 & 6 & 9 & 291 & 291 & 0.485 & 0.211 & 1.16 & M. \\
\hline 29 & 3130 & 198 & 216 & 6 & 9 & 291 & 291 & 0.485 & 0.191 & 1.03 & M. \\
\hline 30 & 1570 & 318 & 216 & 6 & 9 & 291 & 291 & 0.539 & 0.351 & 1.1 & M. \\
\hline 31 & 3130 & 318 & 216 & 6 & 9 & 291 & 291 & 0.539 & 0.314 & 1.07 & M. \\
\hline 32 & 1830 & 198 & 252 & 6 & 9 & 291 & 291 & 0.556 & 0.211 & 1.06 & M. \\
\hline 33 & 1830 & 318 & 252 & 6 & 9 & 291 & 291 & 0.604 & 0.351 & 1.08 & M. \\
\hline 34 & 2090 & 198 & 288 & 6 & 9 & 291 & 291 & 0.628 & 0.211 & 1.0 & M. \\
\hline 35 & 2090 & 318 & 288 & 6 & 9 & 291 & 291 & 0.671 & 0.351 & 1.05 & M. \\
\hline 36 & 1040 & 198 & 144 & 6 & 9 & 523 & 523 & 0.468 & 0.283 & 1.2 & M. \\
\hline 37 & 1040 & 318 & 144 & 6 & 9 & 523 & 523 & 0.565 & 0.470 & 1.07 & M. \\
\hline 38 & 1300 & 198 & 180 & 6 & 9 & 523 & 523 & 0.558 & 0.283 & 1.05 & M. \\
\hline 39 & 2600 & 198 & 180 & 6 & 9 & 523 & 523 & 0.558 & 0.255 & 0.99 & M. \\
\hline 40 & 1300 & 318 & 180 & 6 & 9 & 523 & 523 & 0.641 & 0.471 & 1.04 & M. \\
\hline 41 & 2600 & 318 & 180 & 6 & 9 & 523 & 523 & 0.641 & 0.420 & 1.0 & M. \\
\hline 42 & 1570 & 198 & 216 & 6 & 9 & 523 & 523 & 0.651 & 0.283 & 0.98 & M. \\
\hline 43 & 1570 & 318 & 216 & 6 & 9 & 523 & 523 & 0.723 & 0.470 & 0.98 & M. \\
\hline \multicolumn{12}{|c|}{ Reported by Suzuki et al. $[17,24,26-28]$} \\
\hline 44 & 900 & 300 & 125 & 3.2 & 9 & 274 & 268 & 0.562 & 0.739 & 1.09 & M. \\
\hline 45 & 900 & 300 & 125 & 6 & 9 & 297 & 268 & 0.376 & 0.321 & 1.43 & M. \\
\hline 46 & 1200 & 300 & 125 & 3.2 & 9 & 274 & 268 & 0.562 & 0.663 & 1.15 & M. \\
\hline 47 & 1200 & 300 & 125 & 4.5 & 9 & 286 & 268 & 0.443 & 0.426 & 1.29 & M. \\
\hline 48 & 1200 & 300 & 125 & 6 & 9 & 297 & 268 & 0.376 & 0.300 & 1.28 & M. \\
\hline 49 & 1200 & 300 & 125 & 9 & 9 & 268 & 268 & 0.307 & 0.185 & 1.3 & M. \\
\hline 50 & 1500 & 300 & 125 & 3.2 & 9 & 274 & 268 & 0.562 & 0.616 & 1.09 & M. \\
\hline 51 & 1500 & 300 & 125 & 6 & 9 & 297 & 268 & 0.376 & 0.289 & 1.23 & M. \\
\hline 52 & 1400 & 450 & 125 & 4.5 & 9 & 516 & 268 & 0.793 & 0.700 & 0.93 & M. \\
\hline 53 & 1400 & 450 & 125 & 4.5 & 12 & 516 & 259 & 0.764 & 0.724 & 0.96 & M. \\
\hline 54 & 1400 & 450 & 125 & 4.5 & 15 & 516 & 268 & 0.747 & 0.765 & 0.98 & M. \\
\hline 55 & 1250 & 250 & 125 & 5.8 & 8.5 & 395 & 374 & 0.418 & 0.298 & 1.22 & M. \\
\hline 56 & 1950 & 250 & 125 & 5.6 & 8.3 & 395 & 374 & 0.430 & 0.295 & 1.18 & M. \\
\hline
\end{tabular}


TABle 1: Continued.

\begin{tabular}{|c|c|c|c|c|c|c|c|c|c|c|c|}
\hline Number & $\begin{array}{c}L \\
(\mathrm{~mm})\end{array}$ & $\begin{array}{c}D \\
(\mathrm{~mm})\end{array}$ & $\begin{array}{c}B \\
(\mathrm{~mm})\end{array}$ & $\begin{array}{c}t_{w} \\
(\mathrm{~mm})\end{array}$ & $\begin{array}{c}t_{f} \\
(\mathrm{~mm})\end{array}$ & $\begin{array}{c}s_{w y} \\
(\mathrm{MPa})\end{array}$ & $\begin{array}{c}s_{f y} \\
(\mathrm{MPa})\end{array}$ & $\left(b / t_{f}\right)_{\mathrm{eq}}$ & $S_{w}$ & $\begin{array}{l}\tau_{\max } \\
\text { (test) }\end{array}$ & $\begin{array}{l}\text { Load (M. stands for monotonic load; } \\
\text { C. stands for cyclic load) }\end{array}$ \\
\hline 57 & 1200 & 300 & 100 & 9 & 9 & 384 & 384 & 0.321 & 0.216 & 1.41 & C. \\
\hline 58 & 2100 & 350 & 150 & 6 & 16 & 499 & 449 & 0.463 & 0.456 & 1.37 & M. \\
\hline 59 & 2100 & 350 & 150 & 6 & 12 & 499 & 506 & 0.521 & 0.464 & 1.33 & M. \\
\hline 60 & 2100 & 350 & 150 & 6 & 9 & 499 & 488 & 0.589 & 0.448 & 1.3 & M. \\
\hline 61 & 2400 & 400 & 150 & 6 & 16 & 499 & 449 & 0.521 & 0.513 & 1.33 & M. \\
\hline 62 & 2400 & 400 & 150 & 6 & 12 & 499 & 506 & 0.574 & 0.524 & 1.3 & M. \\
\hline 63 & 1800 & 450 & 200 & 6 & 12 & 415 & 368 & 0.611 & 0.579 & 1.06 & M. \\
\hline 64 & 1800 & 450 & 200 & 6 & 16 & 415 & 377 & 0.558 & 0.628 & 1.14 & M. \\
\hline 65 & 1800 & 450 & 200 & 6 & 19 & 415 & 375 & 0.532 & 0.658 & 1.16 & M. \\
\hline 66 & 1800 & 450 & 200 & 9 & 16 & 383 & 377 & 0.412 & 0.367 & 1.3 & M. \\
\hline 67 & 1800 & 450 & 150 & 6 & 12 & 415 & 368 & 0.565 & 0.539 & 1.11 & M. \\
\hline 68 & 2500 & 450 & 200 & 6 & 16 & 415 & 377 & 0.558 & 0.566 & 1.18 & M. \\
\hline 69 & 1100 & 450 & 150 & 6 & 12 & 415 & 368 & 0.565 & 0.618 & 1.14 & M. \\
\hline 70 & 1600 & 400 & 150 & 6 & 16 & 398 & 376 & 0.467 & 0.520 & 1.3 & M. \\
\hline 71 & 1600 & 400 & 200 & 6 & 16 & 398 & 376 & 0.500 & 0.575 & 1.15 & M. \\
\hline 72 & 1600 & 400 & 150 & 6 & 22 & 398 & 357 & 0.432 & 0.550 & 1.14 & M. \\
\hline 73 & 1200 & 400 & 200 & 6 & 12 & 398 & 382 & 0.562 & 0.591 & 1.1 & M. \\
\hline 74 & 1200 & 400 & 200 & 6 & 16 & 398 & 376 & 0.500 & 0.644 & 1.07 & M. \\
\hline \multicolumn{12}{|c|}{ Reported by Fujikawa and Fujiwara [18] } \\
\hline 75 & 900 & 241 & 150.2 & 4.48 & 8.6 & 344 & 272 & 0.451 & 0.399 & 1.39 & C. \\
\hline 76 & 899 & 248 & 150.4 & 4.56 & 12.16 & 344 & 293 & 0.391 & 0.458 & 1.22 & C. \\
\hline 77 & 1125 & 241 & 149.7 & 4.49 & 9.27 & 344 & 548 & 0.524 & 0.506 & 1.11 & C. \\
\hline 78 & 1260 & 331 & 149.9 & 4.6 & 8.57 & 344 & 272 & 0.541 & 0.485 & 1.29 & C. \\
\hline 79 & 1258 & 338 & 150.4 & 4.38 & 12.13 & 344 & 293 & 0.515 & 0.596 & 1.26 & C. \\
\hline 80 & 1266 & 330 & 150.2 & 4.3 & 9.21 & 344 & 548 & 0.627 & 0.716 & 1.05 & C. \\
\hline 81 & 450 & 242 & 150.1 & 4.32 & 8.61 & 344 & 272 & 0.460 & 0.532 & 1.16 & C. \\
\hline 82 & 462 & 247 & 150.5 & 4.33 & 12.18 & 344 & 293 & 0.403 & 0.526 & 1.24 & C. \\
\hline 83 & 450 & 241 & 150.5 & 4.31 & 9.23 & 344 & 548 & 0.535 & 0.528 & 1.2 & C. \\
\hline 84 & 629 & 330 & 151 & 4.34 & 8.63 & 344 & 272 & 0.561 & 0.666 & 1.16 & C. \\
\hline 85 & 630 & 339 & 150.5 & 4.35 & 12.15 & 344 & 293 & 0.519 & 0.739 & 1.06 & C. \\
\hline 86 & 629 & 332 & 151.7 & 4.37 & 9.28 & 344 & 548 & 0.624 & 0.736 & 1.02 & C. \\
\hline \multicolumn{12}{|c|}{ Reported by Fujiwara and Kato [19] } \\
\hline 87 & 1496 & 244 & 151 & 4.5 & 8.99 & 372 & 282 & 0.457 & 0.353 & 1.25 & C. \\
\hline 88 & 1492 & 250 & 150.4 & 4.5 & 11.99 & 372 & 309 & 0.414 & 0.397 & 1.28 & C. \\
\hline 89 & 1497 & 244 & 150 & 4.4 & 8.7 & 372 & 368 & 0.501 & 0.402 & 1.3 & C. \\
\hline 90 & 1499 & 244 & 149.6 & 4.4 & 8.83 & 372 & 547 & 0.556 & 0.479 & 1.08 & C. \\
\hline \multicolumn{12}{|c|}{ Reported by Yoda et al. [20] } \\
\hline 91 & 1100 & 270.5 & 108.5 & 4.23 & 5.57 & 306 & 310 & 0.529 & 0.400 & 1.18 & M. \\
\hline 92 & 1350 & 314.4 & 108.3 & 4.23 & 5.57 & 306 & 310 & 0.574 & 0.455 & 1.15 & M. \\
\hline 93 & 1600 & 359.7 & 108.8 & 4.23 & 5.57 & 306 & 310 & 0.626 & 0.514 & 1.13 & M. \\
\hline 94 & 1850 & 405.8 & 108 & 4.23 & 5.57 & 306 & 310 & 0.678 & 0.575 & 1.03 & M. \\
\hline 95 & 2100 & 449.6 & 108.9 & 4.23 & 5.57 & 306 & 310 & 0.732 & 0.634 & 1.0 & M. \\
\hline 96 & 2350 & 494.6 & 108.2 & 4.23 & 5.57 & 306 & 310 & 0.786 & 0.696 & 0.91 & M. \\
\hline 97 & 1400 & 269.6 & 144.3 & 4.23 & 5.57 & 306 & 310 & 0.624 & 0.403 & 1.09 & M. \\
\hline 98 & 1650 & 315.5 & 143.8 & 4.23 & 5.57 & 306 & 310 & 0.664 & 0.464 & 1.03 & M. \\
\hline 99 & 1950 & 359.5 & 144.8 & 4.23 & 5.57 & 306 & 310 & 0.709 & 0.522 & 1.03 & M. \\
\hline 100 & 2250 & 405.1 & 144.3 & 4.23 & 5.57 & 306 & 310 & 0.755 & 0.583 & 1.0 & M. \\
\hline 101 & 2550 & 449.2 & 144.5 & 4.23 & 5.57 & 306 & 310 & 0.803 & 0.643 & 0.93 & M. \\
\hline 102 & 2900 & 494.9 & 144.1 & 4.23 & 5.57 & 306 & 310 & 0.854 & 0.706 & 0.93 & M. \\
\hline 103 & 1550 & 270.4 & 180.3 & 4.23 & 5.57 & 306 & 310 & 0.730 & 0.414 & 0.99 & M. \\
\hline 104 & 1850 & 315 & 180.3 & 4.23 & 5.57 & 306 & 310 & 0.764 & 0.471 & 0.97 & M. \\
\hline 105 & 2200 & 360.5 & 179.8 & 4.23 & 5.57 & 306 & 310 & 0.801 & 0.530 & 0.92 & M. \\
\hline 106 & 2550 & 404.8 & 180.4 & 4.23 & 5.57 & 306 & 310 & 0.844 & 0.590 & 0.87 & M. \\
\hline 107 & 2850 & 449.2 & 180.9 & 4.23 & 5.57 & 306 & 310 & 0.888 & 0.651 & 0.84 & M. \\
\hline 108 & 3200 & 494.5 & 180.3 & 4.23 & 5.57 & 306 & 310 & 0.934 & 0.713 & 0.8 & M. \\
\hline 109 & 1799 & 479.6 & 108.1 & 3.23 & 5.89 & 286 & 309 & 0.917 & 0.907 & 0.92 & M. \\
\hline 110 & 1849 & 576.4 & 107.9 & 3.23 & 5.89 & 286 & 309 & 1.080 & 1.083 & 0.82 & M. \\
\hline 111 & 2549 & 479.4 & 144.1 & 3.23 & 5.89 & 286 & 309 & 0.969 & 0.911 & 0.87 & M. \\
\hline
\end{tabular}


TABle 1: Continued.

\begin{tabular}{|c|c|c|c|c|c|c|c|c|c|c|c|}
\hline Number & $\begin{array}{c}L \\
(\mathrm{~mm})\end{array}$ & $\begin{array}{c}D \\
(\mathrm{~mm})\end{array}$ & $\begin{array}{c}B \\
(\mathrm{~mm})\end{array}$ & $\begin{array}{c}t_{w} \\
(\mathrm{~mm})\end{array}$ & $\begin{array}{c}t_{f} \\
(\mathrm{~mm})\end{array}$ & $\begin{array}{c}s_{w y} \\
(\mathrm{MPa})\end{array}$ & $\begin{array}{c}s_{f y} \\
(\mathrm{MPa})\end{array}$ & $\left(b / t_{f}\right)_{\mathrm{eq}}$ & $S_{w}$ & $\begin{array}{c}\tau_{\max } \\
\text { (test) }\end{array}$ & $\begin{array}{l}\text { Load (M. stands for monotonic load; } \\
\text { C. stands for cyclic load) }\end{array}$ \\
\hline 112 & 2603 & 576 & 143.6 & 3.23 & 5.89 & 286 & 309 & 1.124 & 1.089 & 0.8 & M. \\
\hline 113 & 3309 & 479.4 & 179.6 & 3.23 & 5.89 & 286 & 309 & 1.031 & 0.916 & 0.86 & M. \\
\hline 114 & 3448 & 575.8 & 180.2 & 3.23 & 5.89 & 286 & 309 & 1.179 & 1.094 & 0.71 & M. \\
\hline 115 & 1100 & 269.5 & 108.5 & 4.23 & 5.57 & 306 & 310 & 0.528 & 0.398 & 1.18 & M. \\
\hline 116 & 1600 & 359.5 & 108.2 & 4.23 & 5.57 & 306 & 310 & 0.624 & 0.513 & 1.14 & M. \\
\hline 117 & 2101 & 449.3 & 108.1 & 4.23 & 5.57 & 306 & 310 & 0.730 & 0.634 & 1.02 & M. \\
\hline 118 & 1399 & 270.4 & 144.5 & 4.23 & 5.57 & 306 & 310 & 0.625 & 0.405 & 1.08 & M. \\
\hline 119 & 1950 & 360.3 & 144 & 4.23 & 5.57 & 306 & 310 & 0.708 & 0.522 & 1.08 & M. \\
\hline 120 & 2551 & 451.1 & 144 & 4.23 & 5.57 & 306 & 310 & 0.804 & 0.645 & 1.0 & M. \\
\hline 121 & 1550 & 270.5 & 180.3 & 4.23 & 5.57 & 306 & 310 & 0.730 & 0.414 & 1.08 & M. \\
\hline 122 & 2199 & 359.6 & 179 & 4.23 & 5.57 & 306 & 310 & 0.798 & 0.529 & 0.96 & M. \\
\hline 123 & 2850 & 449.5 & 179.5 & 4.23 & 5.57 & 306 & 310 & 0.885 & 0.651 & 0.9 & M. \\
\hline \multicolumn{12}{|c|}{ Reported by Konomi et al. [21] } \\
\hline 124 & 1850 & 450 & 200 & 9 & 12 & 290 & 305 & 0.425 & 0.310 & 1.37 & C. \\
\hline 125 & 1850 & 450 & 200 & 9 & 12 & 290 & 305 & 0.425 & 0.310 & 1.35 & C. \\
\hline 126 & 1850 & 450 & 200 & 9 & 12 & 290 & 305 & 0.425 & 0.310 & 1.33 & C. \\
\hline \multicolumn{12}{|c|}{ Reported by Makishi and Yamamoto et al. $[22,23]$} \\
\hline 127 & 1800 & 506 & 201 & 11 & 19 & 367 & 357 & 0.357 & 0.325 & 1.43 & C. \\
\hline 128 & 1800 & 506 & 201 & 11 & 19 & 367 & 357 & 0.357 & 0.325 & 1.35 & C. \\
\hline 129 & 1800 & 506 & 201 & 11 & 19 & 367 & 357 & 0.357 & 0.325 & 1.38 & C. \\
\hline 130 & 1800 & 506 & 201 & 11 & 19 & 367 & 357 & 0.357 & 0.325 & 1.35 & C. \\
\hline \multicolumn{12}{|c|}{ Reported by Ito et al. [25] } \\
\hline 131 & 1200 & 300 & 130 & 6 & 12 & 403 & 358 & 0.391 & 0.367 & 1.45 & C. \\
\hline 132 & 1200 & 300 & 130 & 6 & 12 & 403 & 358 & 0.391 & 0.367 & 1.51 & C. \\
\hline 133 & 1200 & 300 & 130 & 6 & 12 & 403 & 358 & 0.391 & 0.367 & 1.39 & C. \\
\hline 134 & 1200 & 300 & 130 & 6 & 12 & 403 & 358 & 0.391 & 0.367 & 1.46 & C. \\
\hline \multicolumn{12}{|c|}{ Reported by Minami et al. [29] } \\
\hline 135 & 2150 & 488 & 300 & 11 & 18 & 387 & 387 & 0.457 & 0.342 & 1.56 & C. \\
\hline 136 & 2150 & 488 & 300 & 11 & 18 & 387 & 387 & 0.457 & 0.342 & 1.55 & C. \\
\hline 137 & 2150 & 488 & 300 & 11 & 18 & 387 & 387 & 0.457 & 0.342 & 1.42 & C. \\
\hline \multicolumn{12}{|c|}{ Reported by Wang et al. [30] } \\
\hline 138 & 1400 & 350 & 175 & 3.2 & 12 & 281 & 306 & 0.653 & 1.006 & 0.73 & C. \\
\hline 139 & 1400 & 350 & 175 & 4.5 & 12 & 267 & 306 & 0.496 & 0.604 & 1.13 & C. \\
\hline 140 & 1400 & 350 & 175 & 4.5 & 16 & 267 & 266 & 0.444 & 0.622 & 1.07 & C. \\
\hline 141 & 1400 & 350 & 175 & 4.5 & 19 & 267 & 257 & 0.423 & 0.666 & 0.96 & C. \\
\hline 142 & 1400 & 350 & 175 & 6 & 9 & 332 & 291 & 0.505 & 0.384 & 1.15 & C. \\
\hline 143 & 1400 & 350 & 175 & 6 & 12 & 332 & 306 & 0.443 & 0.417 & 1.21 & C. \\
\hline 144 & 1400 & 350 & 175 & 6 & 16 & 332 & 266 & 0.387 & 0.423 & 1.26 & C. \\
\hline 145 & 1400 & 350 & 175 & 9 & 12 & 291 & 306 & 0.353 & 0.245 & 1.32 & C. \\
\hline 146 & 1050 & 350 & 175 & 4.5 & 12 & 267 & 306 & 0.496 & 0.688 & 0.95 & C. \\
\hline 147 & 1050 & 350 & 175 & 6 & 12 & 332 & 306 & 0.443 & 0.461 & 1.25 & C. \\
\hline \multicolumn{12}{|c|}{ Reported by Kimura [14] } \\
\hline 148 & 1000 & 202 & 150 & 8.2 & 11.9 & 323 & 283 & 0.270 & 0.154 & 1.45 & M. \\
\hline 149 & 1000 & 202 & 150 & 8.1 & 12.1 & 323 & 283 & 0.267 & 0.157 & 1.67 & C. \\
\hline 150 & 1000 & 201 & 150 & 8.25 & 12 & 323 & 283 & 0.268 & 0.152 & 1.53 & C. \\
\hline 151 & 1000 & 201 & 101 & 8.25 & 12 & 323 & 283 & 0.205 & 0.140 & 1.3 & M. \\
\hline 152 & 1000 & 202 & 100 & 8.25 & 12.1 & 323 & 283 & 0.204 & 0.140 & 1.42 & C. \\
\hline 153 & 1000 & 201 & 200 & 8.2 & 11.8 & 323 & 283 & 0.342 & 0.167 & 1.26 & M. \\
\hline 154 & 1000 & 201 & 201 & 8.2 & 11.8 & 323 & 283 & 0.344 & 0.168 & 1.4 & C. \\
\hline 155 & 800 & 202 & 150 & 8.25 & 12 & 323 & 283 & 0.268 & 0.164 & 1.42 & M. \\
\hline 156 & 800 & 202 & 150 & 8.25 & 11.9 & 323 & 283 & 0.270 & 0.164 & 1.52 & C. \\
\hline 157 & 1200 & 201 & 150 & 8.35 & 11.9 & 323 & 283 & 0.269 & 0.143 & 1.41 & M. \\
\hline 158 & 1200 & 201 & 151 & 8.25 & 12 & 323 & 283 & 0.269 & 0.146 & 1.51 & C. \\
\hline
\end{tabular}




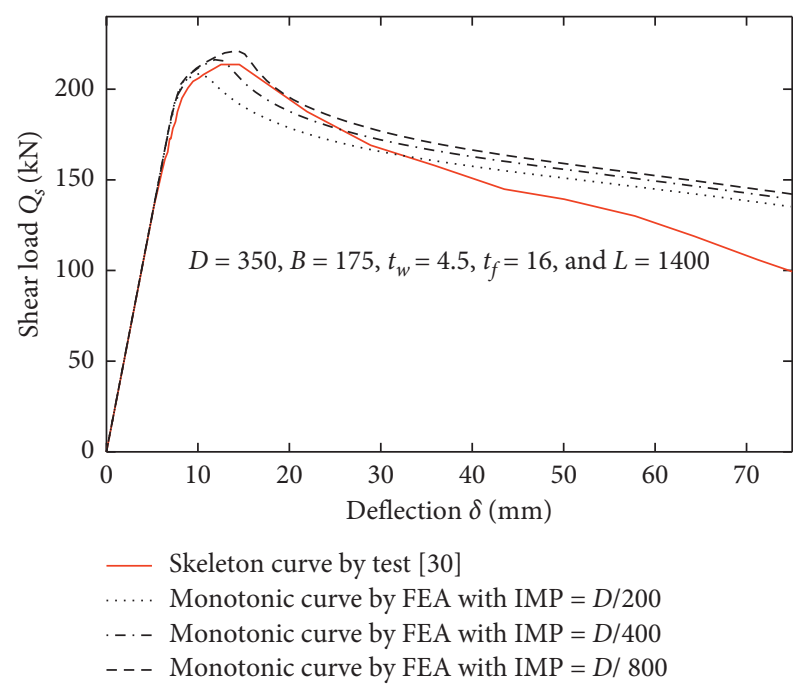

(a)

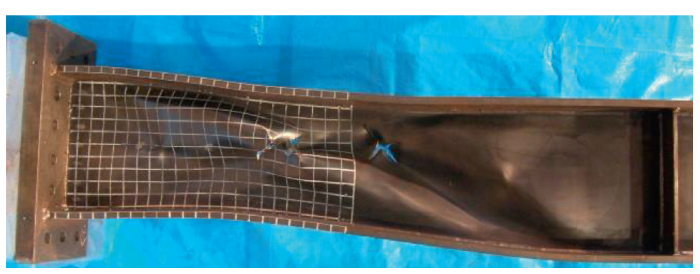

(b)

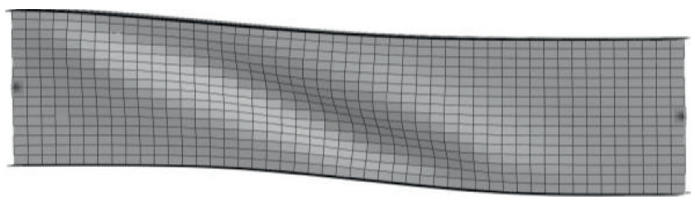

(c)

FIgURE 4: Comparison between FEA and test [30]. (a) Load versus deflection response. (b) Web buckling collapse mode by test [30]. (c) Web buckling collapse mode by FEA with IMP $=D / 400$.

$$
D_{w}=\frac{E t_{w}^{3}}{12\left(1-v^{2}\right)}
$$

where $E$ is Young's modulus and $v$ is the Poisson ratio $(v=0.3) . \sigma_{\text {crw }}$ and $\tau_{\text {crw }}$ in equations (9) and (10) are the critical value of $\sigma \mathrm{b}$ and $\tau s$, which are defined as

$$
\begin{gathered}
\sigma_{\mathrm{crw}}=k_{\mathrm{bw}} \frac{\pi^{2} E}{12\left(1-v^{2}\right)} \frac{1}{\left(d / t_{w}\right)^{2}}, \\
\tau_{\mathrm{crw}}=k_{\mathrm{sw}} \frac{\pi^{2} E}{12\left(1-v^{2}\right)} \frac{1}{\left(d / t_{w}\right)^{2}},
\end{gathered}
$$

where $k_{b w}$ and $k_{s w}$ denote the buckling coefficients due to $\sigma_{\text {crw }}, \tau_{\text {crw }}$, and $k_{b w} / k_{s w}=\alpha$ (equation (6)). The out-of-plane displacement $W$ is expressed by a double Fourier series function as follows:

$$
W=\sum_{m=1}^{M} \sum_{n=1}^{N} e_{m, n} \cdot f_{m}(x) g_{n}(x),
$$

where $e_{m n}$ is the series coefficient and $f_{m}(x)$ and $g_{n}(y)$ are the Fourier series functions. Assuming the edges of web are clamped, the functions $f_{m}(x)$ and $g_{n}(y)$ can be expressed as

$$
\begin{aligned}
& f_{m}(x)=\sin \frac{\pi x}{L} \cdot \sin \frac{m \pi x}{L}, \\
& g_{n}(y)=\sin \frac{\pi y}{d} \cdot \sin \frac{n \pi y}{d} .
\end{aligned}
$$

Here, $W, f_{m}(x)$, and $g_{n}(y)$ in above equations are replaced with $\omega, \mu_{m}(x)$, and $v_{n}(y)$, respectively, as follows:

$$
\begin{aligned}
\omega & =\sum_{m=1}^{M} \sum_{n=1}^{N} e_{m, n} \cdot \mu_{m}(x) \cdot v_{n}(y), \\
\mu_{m}(x) & =\sin \pi x \cdot \sin m \pi x, \\
v_{n}(y) & =\sin \pi y \cdot \sin n \pi y .
\end{aligned}
$$

Equations (8)-(10) can be written as the following equivalent equations:

$$
\begin{gathered}
U=\frac{1}{2} \frac{D_{w}}{d^{2}} \int_{0}^{1} \int_{0}^{1}\left\{\frac{1}{\lambda_{w}^{3}}\left(\frac{\partial^{2} \omega}{\partial x^{2}}\right)^{2}+\lambda_{w}\left(\frac{\partial^{2} \omega}{\partial y^{2}}\right)^{2}+\frac{2 v}{\lambda_{w}} \frac{\partial^{2} \omega}{\partial x^{2}} \frac{\partial^{2} \omega}{\partial y^{2}}\right. \\
\left.+\frac{2(1-v)}{\lambda_{w}}\left(\frac{\partial^{2} \omega}{\partial x \partial y}\right)^{2}\right\} \mathrm{d} x \mathrm{~d} y \\
V_{b}=\frac{1}{2} k_{\mathrm{bw}} \frac{\pi^{2} D_{w}}{d^{2}} \int_{0}^{1} \int_{0}^{1} \frac{(1-\beta x)(1-2 y)}{\lambda_{w}}\left(\frac{\partial \omega}{\partial x}\right)^{2} \mathrm{~d} x \mathrm{~d} y \\
V_{s}=k_{\mathrm{sw}} \frac{\pi^{2} D_{w}}{d^{2}} \int_{0}^{1} \int_{0}^{1} \frac{\partial \omega}{\partial x} \frac{\partial \omega}{\partial y} \mathrm{~d} x \mathrm{~d} y
\end{gathered}
$$

According to the stationary value theory of total potential energy, the conditional expression for the critical state of local stability is 


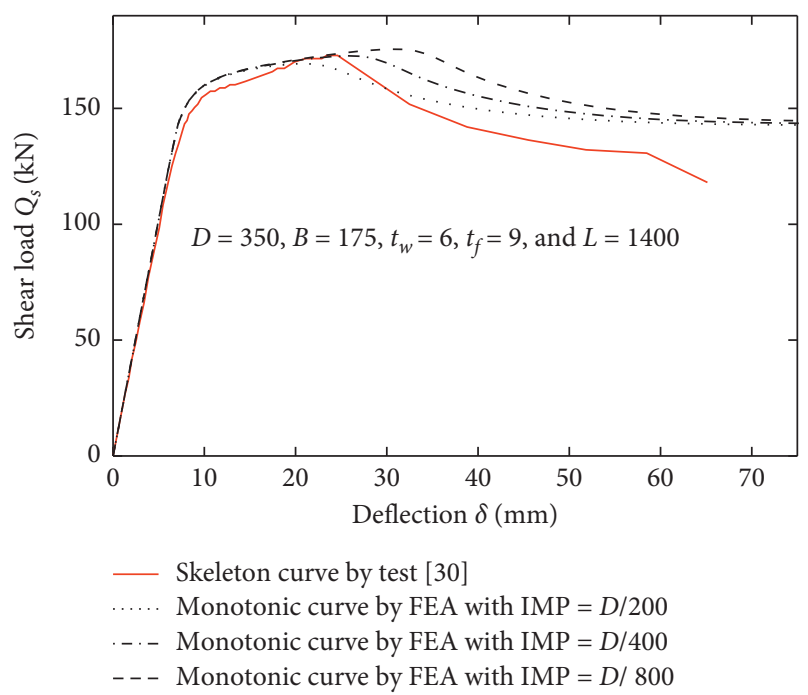

(a)

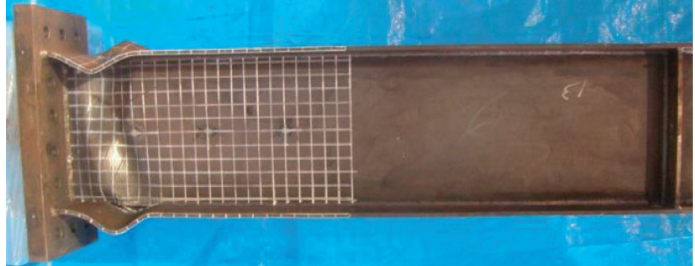

(b)

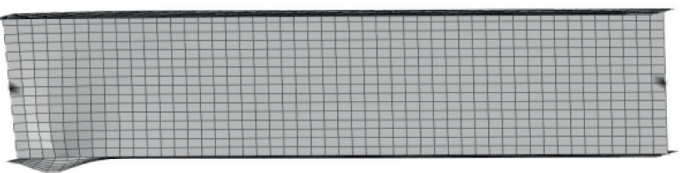

(c)

Figure 5: Comparison between FEA and test [30]. (a)Load versus deflection response. (b) Flange buckling collapse mode by test [30]. (c) Flange buckling collapse mode by FEA with IMP $=D / 400$.

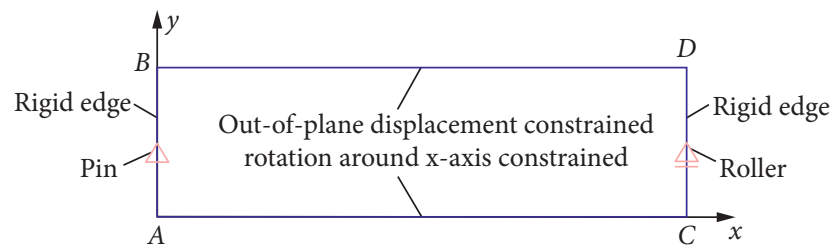

Figure 6: Boundary conditions of web.

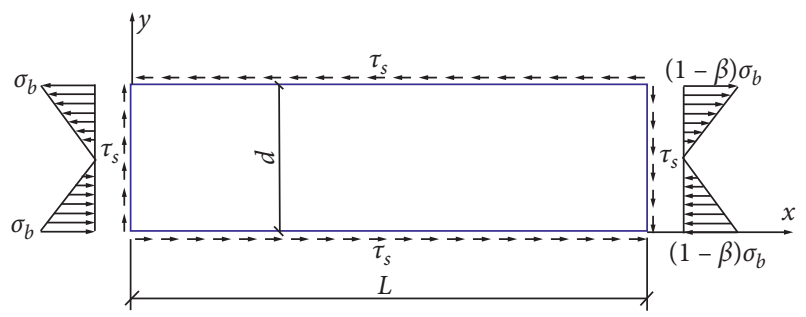

FIgURE 7: Stress distribution of web.

$\frac{\partial \Pi}{\partial e_{i, j}}=\frac{\partial U-\partial V_{b}-\partial V_{s}}{\partial e_{i, j}}=0, \quad(i=1,2, \ldots, M, j=1,2, \ldots, N)$.

To solve the buckling coefficients $k_{\mathrm{bw}}$ and $k_{\mathrm{sw}}$ in equation (20), a generalized eigenvalue analysis is required. The involved parameters to be input are the aspect ratio $\lambda_{w}$, moment gradient $\beta$, and stress ratio $\alpha=k_{\mathrm{sw}} / k_{\mathrm{bw}}$ (equation (6)).
The theoretic analysis using Ritz's energy method is presented above. The energy method using Fourier series functions showed sound convergences, small amount of computations, and high accuracies in the previous studies [9]. Moreover, the energy method has been validated using the finite element analysis (FEA), which showed that the buckling coefficients of a single web with clamped edges gave good agreements with those of an $\mathrm{H}$-section beam web when the rotations of flanges were clamped [12]. However, relevant studies are rather limited towards the condition when flanges are not clamped.

2.3. Elastic Buckling of an H-Section Beam and a Single Web. When the out-of-plane displacement of flanges is constrained, the flange buckling will be prevented to allow web buckling to occur only. For a normal H-section beam, the lower flange (in compression) is not constrained, and the web buckling and flange buckling may occur simultaneously. In the following finite element analysis (FEA), two cases of boundary conditions are considered: Case 1: the out-ofplane displacement of flanges which is constrained (clamped flanges); Case 2: flanges without any constraint (free flanges).

The elastic buckling analysis results of $\mathrm{H}$-section beams obtained by FEA and the elastic buckling analysis results of single webs obtained by theoretical method are shown in Figure 8 . The $\mathrm{H}$-section beams with constant dimension of $L=2400 \mathrm{~mm}, \beta=1, d=D=400 \mathrm{~mm}$ (due to the shell elements, $d=D), t_{w}=4 \mathrm{~mm}, b=150 \mathrm{~mm}$, and variable dimension of $t_{f}$ in the range of $5 \mathrm{~mm} \leq t_{f} \leq 15 \mathrm{~mm}$ are analyzed by FEA, where $L / D=2400 / 400=6$ and $L / B=2400 /$ 


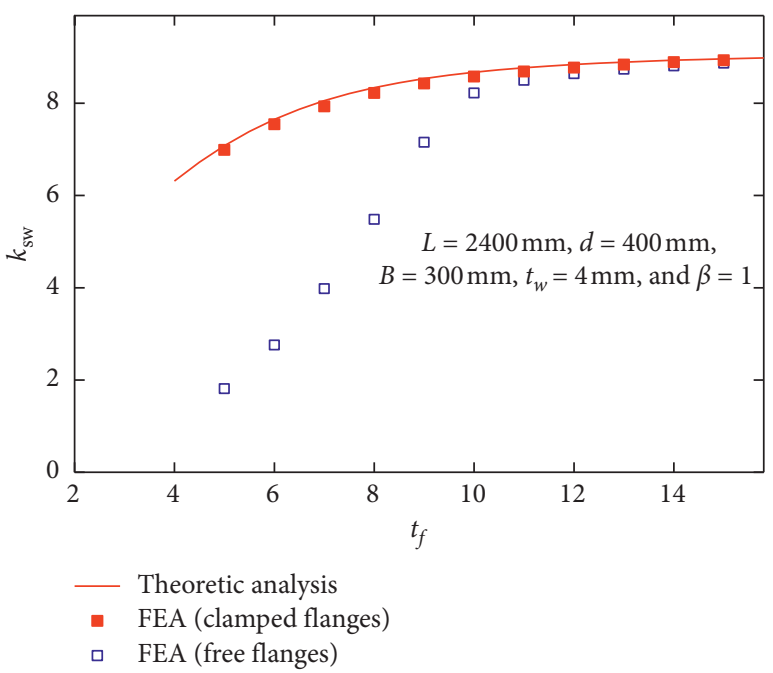

FIGURE 8: $k_{\mathrm{sw}}$ versus $t_{f}$ by different methods.

$300=8<16$. The single webs with the same shape and stress condition (using equation (6)) are analyzed as well by the theoretical energy method presented above. It is shown that the shear buckling coefficient $k_{\mathrm{sw}}$ of $\mathrm{H}$-section beams with clamped flanges (Case 1) obtained by FEA is close to the coefficient $k_{\mathrm{sw}}$ of single webs obtained by theoretical energy method, which corresponds to the previous research [12]. When the flanges are not clamped (Case 2: free flanges), the coefficient $k_{\text {sw }}$ of $\mathrm{H}$-section beams with $t_{f}>10 \mathrm{~mm}$ obtained by FEA is close to the coefficient $k_{\mathrm{sw}}$ of single webs as well. However, when $t_{f}<10 \mathrm{~mm}$, the $k_{\mathrm{sw}}$ of $\mathrm{H}$-section beams are smaller than $k_{s w}$ of single webs due to the effect of flange buckling.

For comparison, three typical buckling modes of $\mathrm{H}$-section beams obtained by FEA with free flanges are given in Figure 9, and three typical buckling modes of single webs obtained by theoretical analysis are given in Figure 10. The theoretical buckling mode is expressed by equation (14), where $e_{m, n}(m=1,2, \ldots, 20 ; n=1,2, \ldots, 10)$ is the first-order eigenvector. By applying the Wolfram Mathematica program, the web buckling modes expressed by equation (14) can be drawn as shown in Figure 10.
As shown in Figure 9(a), the buckling mode of an $\mathrm{H}$-section beam (with $t_{f}=12 \mathrm{~mm}$ and free flanges) is controlled by web buckling, in which the web buckling mode is basically the same as the buckling mode (Figure 10(a)) of a single web with identical shape and stress condition $\left(\lambda_{w}=2400 / 400=6, \beta=1\right.$, and $\left.\alpha=0.4028\right)$, and the shear buckling coefficients are close to each other $(8.64 \approx 8.84)$.

As shown in Figure 9(b), even with a slightly buckled flange, the local buckling of an $\mathrm{H}$-section beam (with $t_{f}=10 \mathrm{~mm}$ and free flanges) is dominated by web buckling, and the buckling mode of the beam web is similar to the buckling mode (Figure 10(b)) of a single web with identical shape and stress condition $\left(\lambda_{w}=6, \beta=1\right.$, and $\left.\alpha=0.3403\right)$, and the shear buckling coefficients are close to each other $(8.22 \approx 8.67)$, which corroborate that the edge condition of a web can be regarded as clamped condition when the local buckling of an $\mathrm{H}$-section beam is dominated by web buckling.

However, when the local buckling of an $\mathrm{H}$-section beam (with $t_{f}=6 \mathrm{~mm}$ and free flanges) is dominated by flange buckling, the buckling mode (Figure 9(c)) of the beam web is different from a single web buckling mode (Figure 10(c)) with identical shape and stress condition $\left(\lambda_{w}=6, \beta=1\right.$, and $\alpha=0.2153)$.

Through comparisons, it is concluded that the buckling of an $\mathrm{H}$-section beam with free flanges is dominated by web buckling when the flange buckling does not occur or slightly occur, and the boundary condition of the longer web edges is close to clamped condition. Therefore, to calculate the elastic local buckling stress of an $\mathrm{H}$-section beam dominated by web buckling, the analytic model can be simplified as a single web with the boundary condition as shown in Figure 6 and with the stress condition as shown in Figure 7. The presented theoretical analytic method with high accuracy and small amount of computations is valuable for studying the buckling stress of an $\mathrm{H}$-section beam web.

\section{Parametric Study}

3.1. Previous Study. Based on theoretic analyses, Suzuki and Ikarashi [12] proposed approximate equations for the buckling coefficients of web with clamped edges as follows:

$$
\begin{aligned}
& k_{\mathrm{sw}}=\left\{\begin{array}{l}
8.98+\frac{5.6}{\lambda_{w}^{2}}, \quad\left(\lambda_{w} \leq \Lambda\right), \\
{\left[R\left(\frac{1}{\lambda_{w}}\right)^{3}-\left(\Lambda^{2}+\frac{2 R}{\Lambda}\right)\left(\frac{1}{\lambda_{w}}\right)^{2}+\left(2 \Lambda+\frac{R}{\Lambda^{2}}\right)\left(\frac{1}{\lambda_{w}}\right)\right] k_{\mathrm{sw} 0}, \quad\left(\lambda_{w}>\Lambda\right),}
\end{array}\right. \\
& k_{\mathrm{bw}}=\frac{k_{\mathrm{sw}}}{\alpha},
\end{aligned}
$$




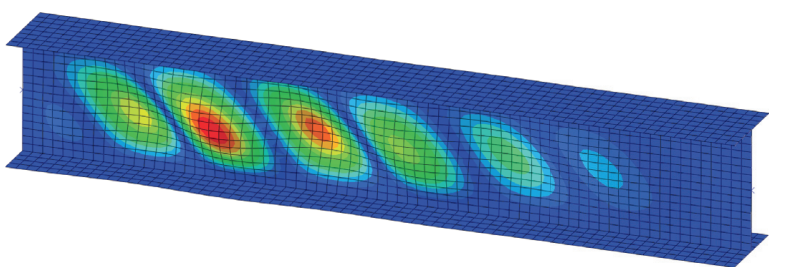

(a)

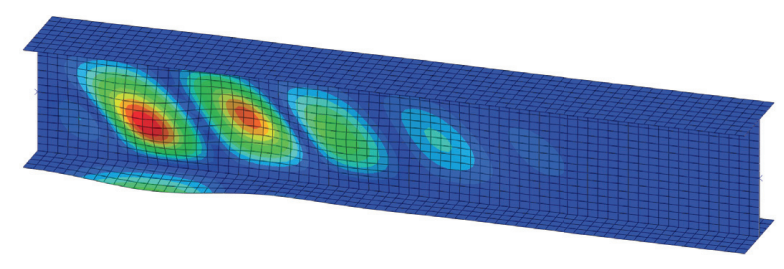

(b)

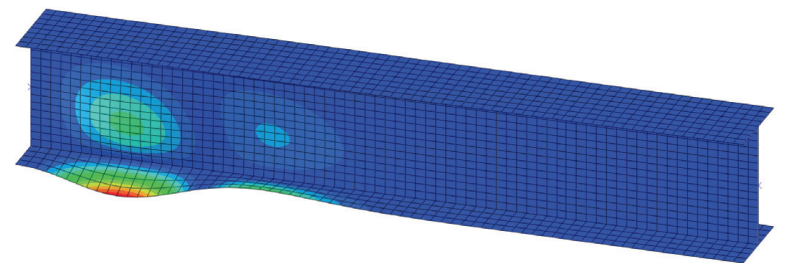

(c)

FIGURE 9: Buckling mode of H-section beam with different $t_{f}$. (a) $L=2400, \beta=1, d=400, t_{w}=4, b=150$, and $t_{f}=12$. (b) $L=2400, \beta=1$, $d=400, t_{w}=4, b=150$, and $t_{f}=10$. (c) $L=2400, \beta=1, d=400, t_{w}=4, b=150$, and $t_{f}=6$.

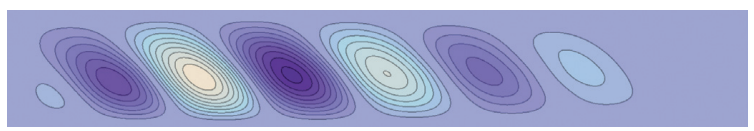

(a)

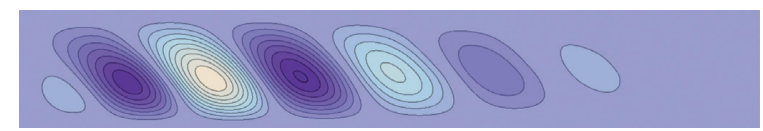

(b)

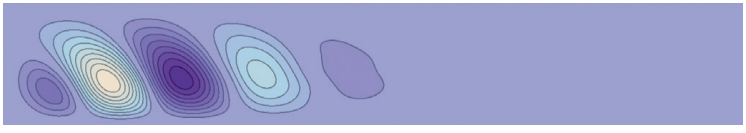

(c)

Figure 10: Buckling mode of web with different $\alpha$. (a) $\lambda_{w}=6, \beta=1$, and $\alpha=0.4028$. (b) $\lambda_{w}=6$, $\beta=1$, and $\alpha=0.3403$. (c) $\lambda_{w}=6$, $\beta=1$, and $\alpha=0.2153$.

where

$$
\begin{aligned}
\eta & =1 / 6+\frac{A_{f}}{A_{w}}, \\
\eta^{*} & =\frac{\eta \beta}{2} \\
\Lambda & =2.1 \eta^{*}+1.7 \\
R & =107 \eta^{* 2}-20.9 \eta^{*}-7.22, \\
\alpha & =\frac{\eta \beta}{\lambda_{w}}
\end{aligned}
$$

As indicated by Suzuki and Ikarashi [12], the proposed equations are only applicable when $1<\beta \leq 2$, and the equations are too complex to be applied for practical uses. These defects should be annihilated. In this study, a new method based on parametric studies is proposed to simplify the calculation method and to expand the application range. The first study is to formulate the equation under simple stress condition such as pure shear, uniform bending, and unequal bending. The interaction between the buckling coefficients of shear and bending is then studied to suggest an approximate formula for the calculation of buckling stress.
3.2. Buckling Coefficient of Web under Pure Shear Force. Let $k_{\mathrm{bw}}=0$ (without considering the effect of bending stress); the critical conditional expression equation (20) can be written as follows:

$$
\frac{\partial \Pi}{\partial e_{i, j}}=\frac{\partial U-\partial V_{s}}{\partial e_{i, j}}=0, \quad(i=1,2, \ldots, M, j=1,2, \ldots, N) .
$$

According to equations (17), (19), and (24), the shear buckling coefficient $k_{s w 0}$ is related to aspect ratio $\lambda_{w}$ only. Result (Figure 11) shows that $k_{\mathrm{sw} 0}$ converges to 8.98 in the case of the infinitely long web, which agrees well with previous research [2]. For the finite length web, when $\lambda_{w}$ is larger than 1, the analyzed result corresponds to the approximate equation suggested by Moheit [3] as follows:

$$
k_{\mathrm{sw} 0}=8.98+\frac{5.6}{\lambda_{w}^{2}} \text {. }
$$

3.3. Buckling Coefficient of Web under Unequal Bending Moment. Let $k_{\mathrm{sw}}=0$ (without considering the effect of shear stress); the critical conditional expression equation (20) can be written as follows: 


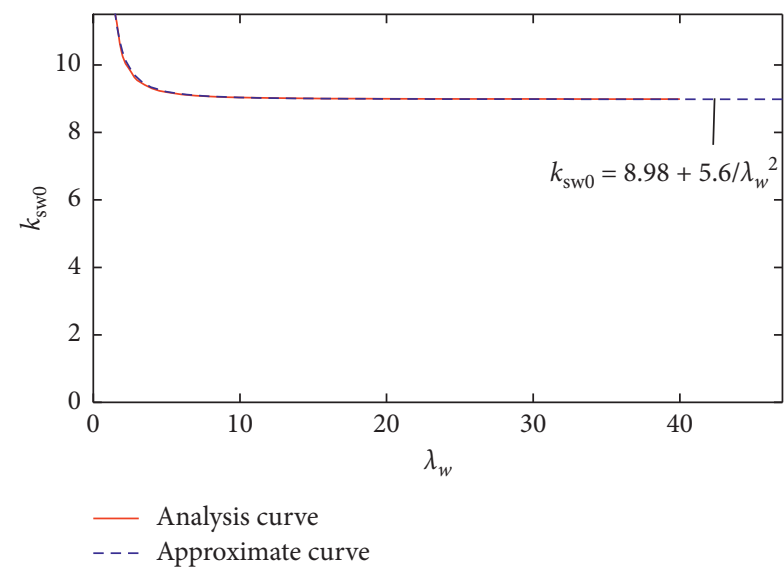

Figure 11: $k_{\mathrm{sw} 0}$ versus $\lambda_{w}$ curves.

$\frac{\partial \Pi}{\partial e_{i, j}}=\frac{\partial U-\partial V_{b}}{\partial e_{i, j}}=0 \quad(i=1,2, \ldots, M, j=1,2, \ldots, N)$.

According to equations (17), (18), and (26), the bending buckling coefficient $k_{\mathrm{bw} 0}$ is related to $\lambda_{w}$ and $\beta$. According to Bijlaard' research [4], for an infinitely long plate under uniform bending $(\beta=0)$, the buckling coefficient $k_{\mathrm{bw} 0}$ was 39.6. In this study, the analyzed results (Figure 12) show that $k_{b w 0}$ converges to 39.6 , which shows a good agreement with Bijlaard' research [4]. $k_{\mathrm{bw} 0}$ is only slightly larger than the lower limit 39.6 when $\lambda_{w} \geq 1$. As shown in Figure 13, when $\beta>0$, the larger value of $\beta$ is, the higher value of $k_{\mathrm{bw} 0}$ is. By changing the abscissa of Figure 13 into $\beta / \lambda_{w}$, Figure 14 can be obtained. It is shown that the curves of $k_{\mathrm{bw} 0}$ versus $\beta / \lambda_{w}$ with various $\beta$ almost overlap with each other, and they can be approximated by the following equation:

$$
k_{\mathrm{bw} 0}=39.6+\frac{40 \beta}{\lambda_{w}} \text {. }
$$

3.4. Interaction Curve of the Buckling Coefficients under Combined Bending and Shear Force. For an H-section beam web, the combined bending and shear stress must be considered. To investigate the interaction between bending and shear stress, $k_{\mathrm{sw}}$ versus $\lambda_{w}$ curves and $k_{\mathrm{bw}}$ versus $\lambda_{w}$ curves with $\beta=2$ and various $A_{f} / A_{w}$ are analyzed based on the critical conditional expression equation (20). As shown in Figure $15, k_{\mathrm{sw}}$ versus $\lambda_{w}$ curves obtained by considering the combined bending and shear stress are lower than $k_{\mathrm{sw} 0}$ versus $\lambda_{w}$ curve obtained by considering the shear stress only. When $\lambda_{w}$ is small enough, all $k_{\mathrm{sw}}$ versus $\lambda_{w}$ curves converge to $k_{\mathrm{sw} 0}$ versus $\lambda_{w}$ curve. As shown in Figure $16, k_{\mathrm{bw}}$ versus $\lambda_{w}$ curves obtained by considering the combined bending and shear stress are lower than $k_{\mathrm{bw} 0}$ versus $\lambda_{w}$ curve obtained by considering the bending stress only. When $\lambda_{w}$ is large enough, all $k_{\mathrm{bw}}$ versus $\lambda_{w}$ curves converge to $k_{\mathrm{bw} 0}$ versus $\lambda_{w}$ curve.

The beams may present various buckling modes due to the different configurations [32, 33]. According to the

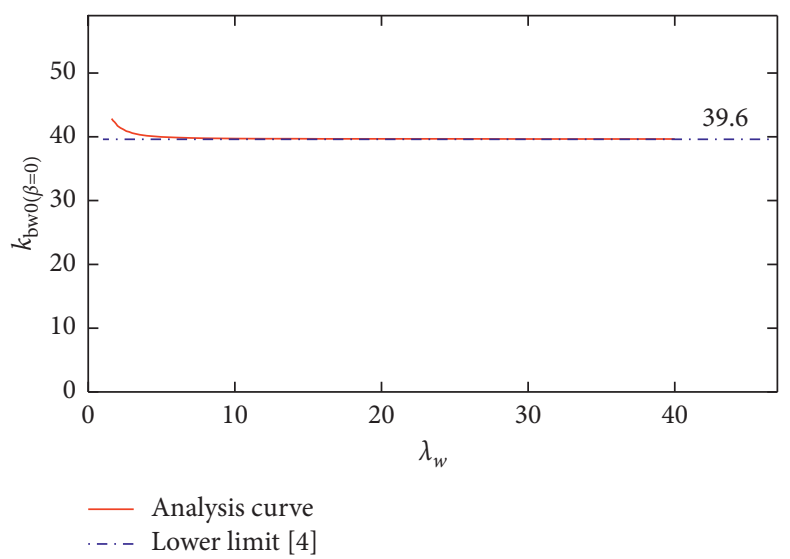

FIgURE 12: $k_{\mathrm{bw0}}$ versus $\lambda_{w}$ curves with $\beta=0$.

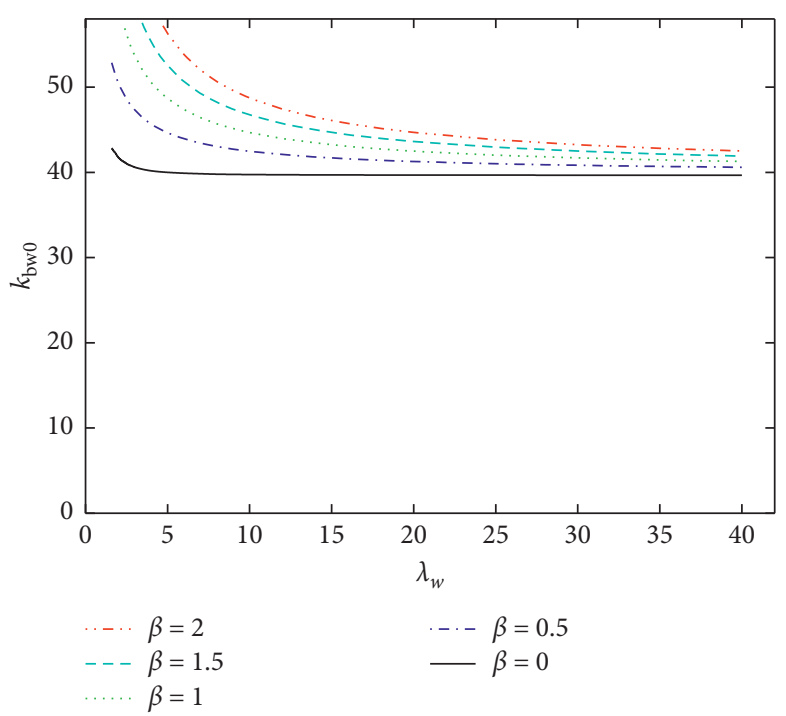

FIgURE 13: $k_{\mathrm{bw} 0}$ versus $\lambda_{w}$ curves with various $\beta$.

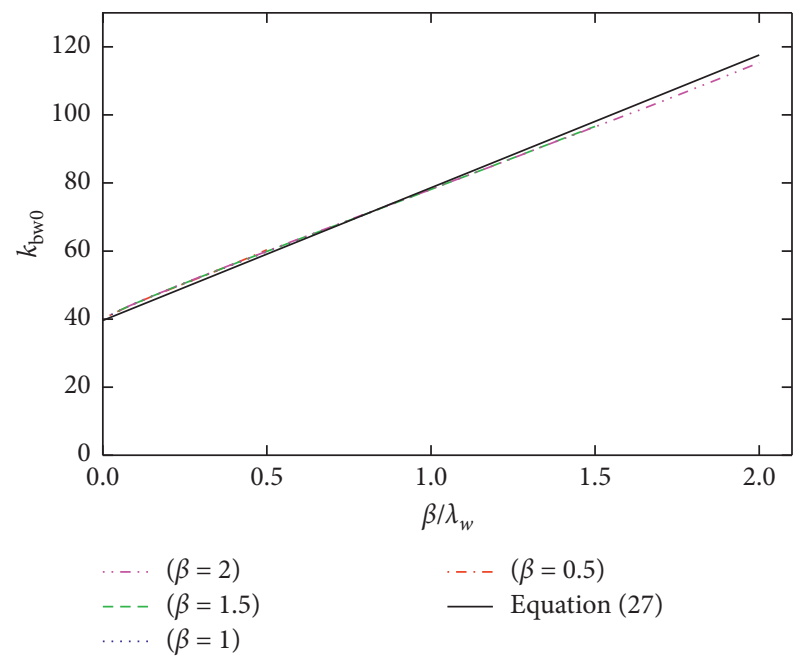

Figure 14: $k_{\mathrm{bw} 0}$ versus $\beta / \lambda_{w}$ curves with various $\beta$. 


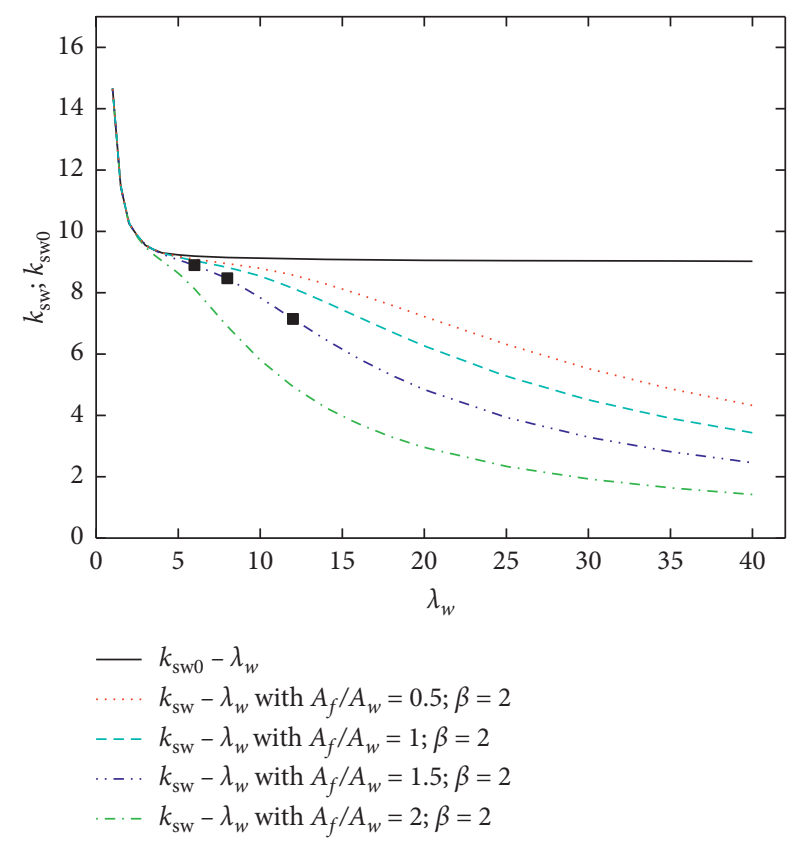

FIgURE 15: $k_{\mathrm{sw}}$ versus $\lambda_{w}$ curves with $\beta=2$ and various $A_{f} / A_{w}$.

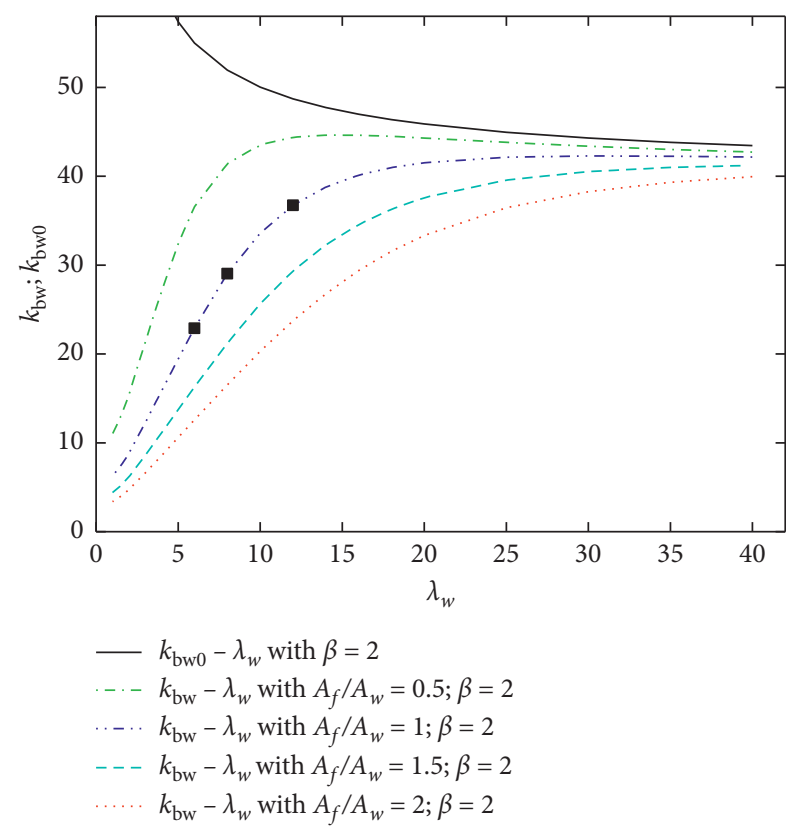

Figure 16: $k_{\mathrm{bw}}$ versus $\lambda_{w}$ curves with $\beta=2$ and various $A_{f} / A_{w}$.

theoretical analyzed results in this study, the elastic local buckling modes of a single web can be roughly divided into three types (shear type, bending type, and intermediate type). Three web buckling modes with $A_{f} / A_{w}=1$ and $\beta=2$ and different aspect ratio $\left(\lambda_{w}=6,8\right.$, and 12) are shown in Figure 17; the symbols of $k_{\mathrm{sw}}$ versus $\lambda_{w}$ and $k_{\mathrm{bw}}$ versus $\lambda_{w}$ are shown in Figures 15 and 16, respectively. The web with $\lambda_{w}=$ 6 presents shear type buckling mode (Figure 17(a)) due to the relatively large shear stress, in which the similar shapes of buckling waves are observed along the length direction. The web with $\lambda_{w}=12$ presents bending type buckling mode
(Figure 17(c)) due to the relatively large bending stress, in which the buckling waves concentrate close to the web ends. The web with $\lambda_{w}=8$ presents intermediate bucking mode when the effects of shear and bending stress are comparable (Figure 17(b)). For all webs, the buckling coefficients $k_{\mathrm{sw}}$ are always smaller than $k_{\mathrm{sw} 0}$ and the buckling coefficients $k_{\mathrm{bw}}$ are always smaller than $k_{\mathrm{bw} 0}$.

As above, $k_{\mathrm{sw} 0}$ and $k_{\mathrm{bw} 0}$ can be regarded as the upper limits of $k_{\mathrm{sw}}$ and $k_{\mathrm{bw}}$, respectively. By taking the ordinate as $k_{\mathrm{sw}} / k_{\mathrm{sw} 0}$ and taking the abscissa as $k_{\mathrm{bw}} / k_{\mathrm{bw} 0}$, Figures 15 and 16 can be expressed by Figure 18. It is shown that all the interaction curves (with $\beta=2$ and various $A_{f} / A_{w}$ ) overlap with each other, and they can be evaluated by an approximate equation as follows:

$$
\left(\frac{k_{\mathrm{bw}}}{k_{\mathrm{bw} 0}}\right)^{2.5}+\left(\frac{k_{\mathrm{sw}}}{k_{\mathrm{sw} 0}}\right)^{2.5}=1
$$

Figure 19 shows a large number of analytical data with various $\lambda_{w}, \beta$, and $A_{f} / A_{w}$ in the range of $1 \leq \lambda_{w} \leq 40$, $0 \leq \beta \leq 2$, and $0.3 \leq A_{f} / A_{w} \leq 2.5$; generally, results of $k_{\mathrm{bw}} / k_{\mathrm{bw} 0}$ versus $k_{\mathrm{sw}} / k_{\mathrm{sw} 0}$ distribute around the curve of equation (28). Therefore, equation (28) can be regarded as an interaction formula to calculate $k_{\mathrm{bw}}$ and $k_{\mathrm{sw}}$.

3.5. Improved Equations. Taking $k_{\mathrm{sw}} / k_{\mathrm{bw}}=a$ and substituting equations (25) and (27) into the interaction formula equation (28), the approximate equations for $k_{\mathrm{bw}}$ and $k_{\mathrm{sw}}$ are obtained as follows:

$$
\begin{gathered}
k_{\mathrm{bw}}=\left\{\frac{1}{\left(39.6+\left(40 \beta / \lambda_{w}\right)\right)^{2.5}}+\left[\frac{\alpha}{8.98+\left(5.6 / \lambda_{w}^{2}\right)}\right]^{2.5}\right\}^{(-1 / 2.5)}, \\
k_{\mathrm{sw}}=k_{\mathrm{bw}} \alpha
\end{gathered}
$$

where $\alpha$ is the stress ratio as shown in equation (6).

The proposed equations (equations (29) and (30)) are far simpler than the previous ones (equation (21) and (22)). For verification and comparison, the proposed curves and FEA results with various cases of $A_{f} / A_{w}$ and $\beta$ are shown in Figures 20-23. It is found that equation (21) gives good agreements with the FEA results when $\beta=2$ and $\beta=1$, as shown in Figures 20 and 21 . However, when $\beta=0.5$ and $\beta=0.1$, equation (21) does not agree with FEA as shown in Figures 22 and 23. As indicated previously, the equations (equations (21) and (22)) can only apply to the range of $1 \leq \beta \leq 2$. This defect is overridden in this study. As shown in Figures 20-23, equation (30) gives good agreements with the FEA results for all cases. The proposed equations with high accuracies are applicable for the full range $(0 \leq \beta \leq 2)$, which are valuable for further studies.

\section{Design Equation for the Ultimate Strength}

In the following research, 158 sets of experimental data [14-30] of $\mathrm{H}$-section cantilever beams (with $\beta=1$ ) as shown in Table 1 are collected to investigate the ultimate strength. 


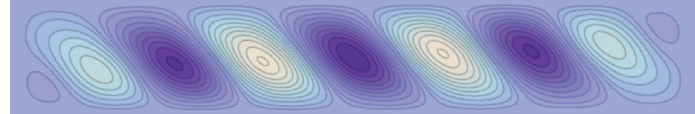

(a)

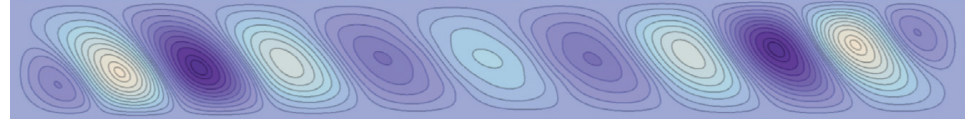

(b)

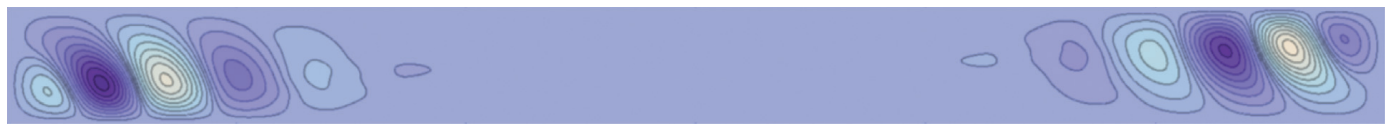

(c)

Figure 17: Different types of web buckling modes. (a) Shear type web buckling mode $\left(\lambda_{w}=6, A_{f} / A_{w}=1\right.$, and $\left.\beta=2\right)$. (b) Intermediate type web buckling mode $\left(\lambda_{w}=8, A_{f} / A_{w}=1\right.$, and $\left.\beta=2\right)$. (c) Bending type web buckling mode $\left(\lambda_{w}=12, A_{f} / A_{w}=1\right.$, and $\left.\beta=2\right)$.
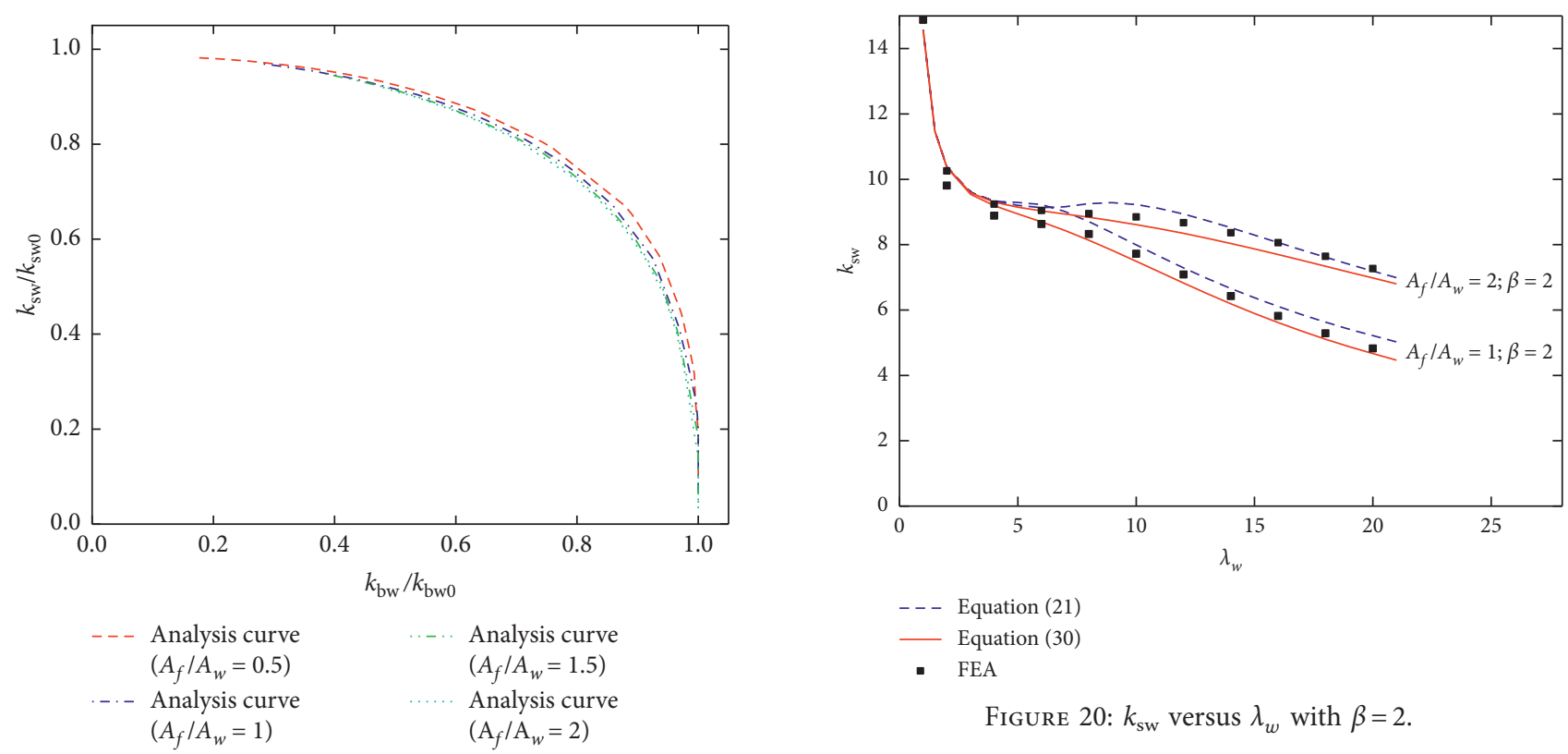

FIGURE 20: $k_{\mathrm{sw}}$ versus $\lambda_{w}$ with $\beta=2$.

FIGURE 18: $k_{\mathrm{bw}} / k_{\mathrm{bw} 0}$ versus $k_{\mathrm{sw}} / k_{\mathrm{sw} 0}$ curves with $\beta=2$ and various $A_{f} / A_{w}$.

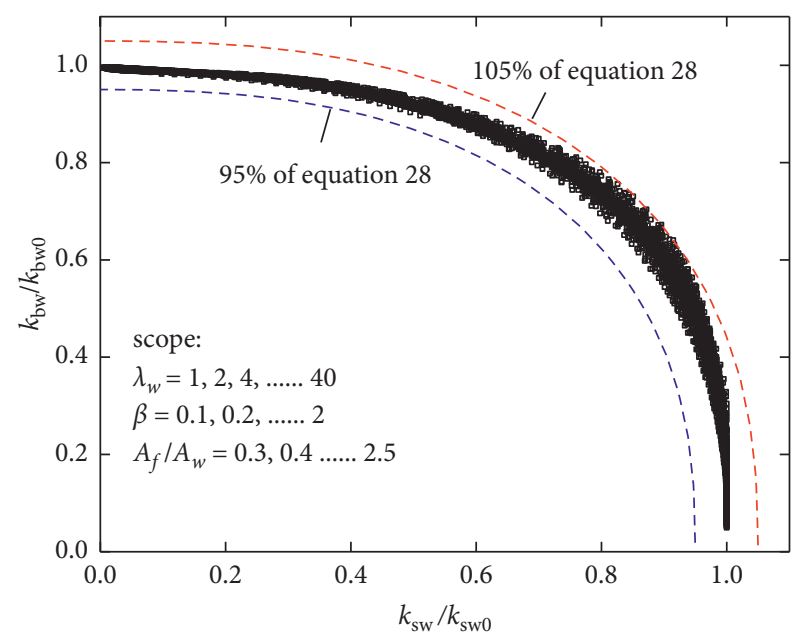

- Analyzed data

Figure 19: $k_{\mathrm{bw}} / k_{\mathrm{bw} 0}$ versus $k_{\mathrm{sw}} / k_{\mathrm{sw} 0}$ plots with various $\beta$ and $A_{f} / A_{w}$.

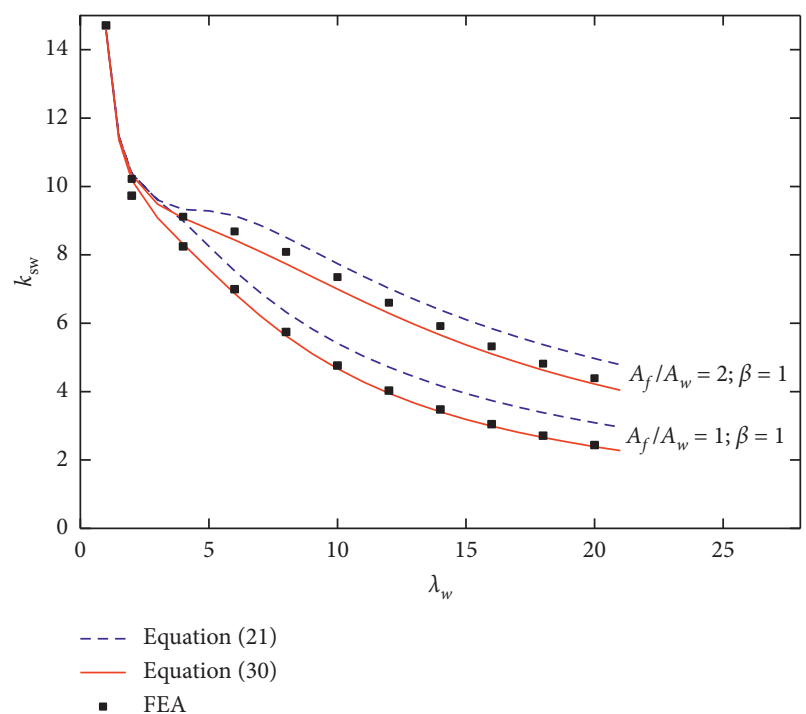

Figure 21: $k_{\mathrm{sw}}$ versus $\lambda_{w}$ with $\beta=1$. 


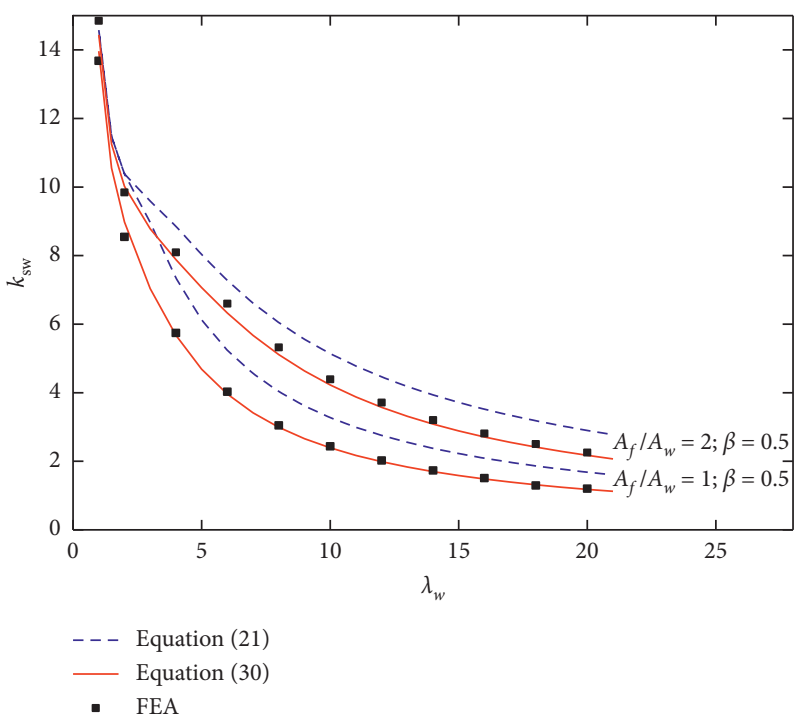

FIgURE 22: $k_{\mathrm{sw}}$ versus $\lambda_{w}$ with $\beta=0.5$.

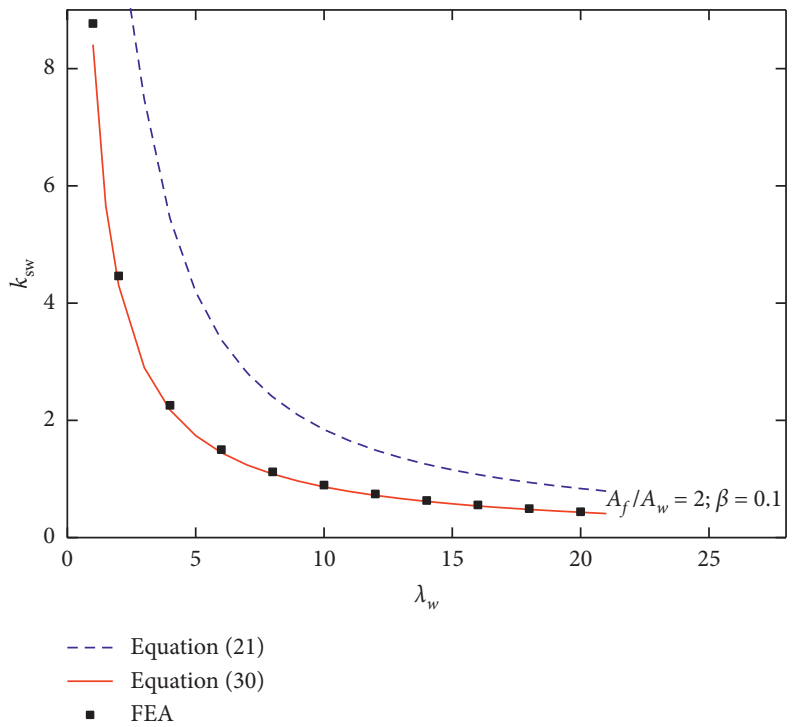

Figure 23: $k_{\mathrm{sw}}$ versus $\lambda_{w}$ with $\beta=0.1$.

All the beams are welded H-section nonscallop beams. Here, the normalized ultimate strength $\tau_{\max }$ is defined as

$$
\tau_{\max }= \begin{cases}\frac{M_{u}}{M_{p}}, & \left(Q_{p} \leq{ }_{w} Q_{p}\right), \\ \frac{Q_{u}}{{ }_{w} Q_{p}}, & \left(Q_{p}>{ }_{w} Q_{p}\right),\end{cases}
$$

where $M_{u}$ and $Q_{u}$ are the ultimate bending strength and shear strength. $M_{p}$ is the full plastic bending moment, $Q_{p}$ is the shear strength in the full plastic bending state, and ${ }_{w} Q_{p}$ is the yield shear strength. $M_{p}, Q_{p}$, and ${ }_{w} Q_{p}$ are expressed as follows:

$$
\begin{aligned}
M_{p} & =\sigma_{\mathrm{fy}} B t_{f} d+\sigma_{\mathrm{wy}} t_{w} \frac{\left(d-t_{f}\right)^{2}}{4}, \\
Q_{p} & =\frac{M_{p} \beta}{L}, \\
{ }_{w} Q_{p} & =\frac{\left(d-t_{f}\right) t_{w} \sigma_{\mathrm{wy}}}{\sqrt{3}} .
\end{aligned}
$$

\subsection{Equivalent Width-Thickness Ratio and Previous Design} Equation. When the width-thickness ratio of flange is large, the buckling of an $\mathrm{H}$-section beam may be dominated by flange buckling. The elastic buckling stress of a long flange (assuming no restraint from the web) under compressive force is expressed as follows [5]:

$$
\sigma_{\mathrm{crf}}=0.425 \frac{\pi^{2} E}{12\left(1-v^{2}\right)} \cdot \frac{1}{\left(b / t_{f}\right)^{2}} .
$$

According to previous studies $[13,14]$, the equivalent width-thickness ratio $\left(b / t_{f}\right)_{\text {eq }}$ can be written as follows:

$$
\left(\frac{b}{t_{f}}\right)_{\mathrm{eq}}=\sqrt{\frac{\sigma_{\mathrm{fy}}}{E}\left(\frac{b}{t_{f}}\right)^{2}+\frac{\sigma_{\mathrm{wy}}}{41 E}\left(\frac{d}{t_{w}}\right)^{2}} .
$$

By taking the ordinate as the normalized ultimate strength $\tau_{\max }$ (obtained from test data) and taking the abscissa as the equivalent width-thickness ratio $\left(b / t_{f}\right)_{\text {eq }}$, Figure 24 can be obtained. There are 94 test data in the range of $\sigma_{\mathrm{crw}}>1.5 \sigma_{\mathrm{crf}}$ and 64 test data in the range of $\sigma_{\mathrm{crw}} \leq 1.5 \sigma_{\mathrm{crf}}$, in which $\sigma_{\text {crw }}$ is calculated by using equations (12) and (29) and $\sigma_{\text {crf }}$ is calculated by equation (35). It is shown that $\tau_{\max }$ has a strong correlation with $\left(b / t_{f}\right)_{\mathrm{eq}}$, and it is reasonable to suppose that the local buckling is dominated by flange buckling when $\sigma_{\text {crw }}>1.5 \sigma_{\text {crf }}$. The calculation of $\tau_{\max }$ has been suggested by Kimura [14] for the flange buckling dominant $\mathrm{H}$-section beams, and $\tau_{\max }$ of the beams under monotonic loads can be expressed as follows:

$$
\tau_{\max }=1.5-0.57\left(\frac{b}{t_{f}}\right)_{\mathrm{eq}}-\frac{0.01 L}{D} .
$$

By taking the ordinate as $\tau_{\max }+0.01 L / D$ and the abscissa as $\left(b / t_{f}\right)_{\mathrm{eq}}$ in the range of $\sigma_{\mathrm{crw}}>1.5 \sigma_{\mathrm{crf}}$, Figure 25 can be obtained, in which equation (37) gives a good agreement with test results. Moreover, the distribution of $\tau_{\max }$ obtained by cyclic tests is slightly higher than that obtained by monotonic tests as indicated by Kimura [14]. However, the correlation between $\tau_{\max }$ and $\left(b / t_{f}\right)_{\text {eq }}$ is not strong when $\sigma_{\text {crw }} \leq 1.5 \sigma_{\text {crf }}$, due to the effect of web buckling as shown in Figure 24. As demonstrated in equation (36), (b/t $\left.t_{f}\right)_{\mathrm{eq}}$ contains the width-thickness ratio of flange and the depththickness ratio of web only. Thus, it is insufficient to regard $\left(b / t_{f}\right)_{\mathrm{eq}}$ as the major parameter to evaluate the ultimate strength when the buckling is dominated by web buckling. 


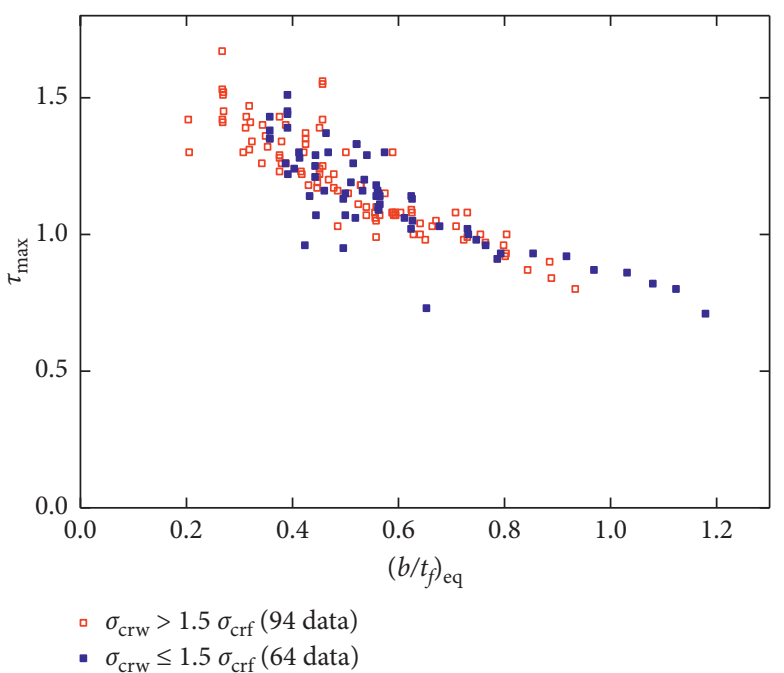

FIgURE 24: $\tau_{\max }$ versus $\left(\mathrm{b} / t_{f}\right)_{\mathrm{eq}}$.

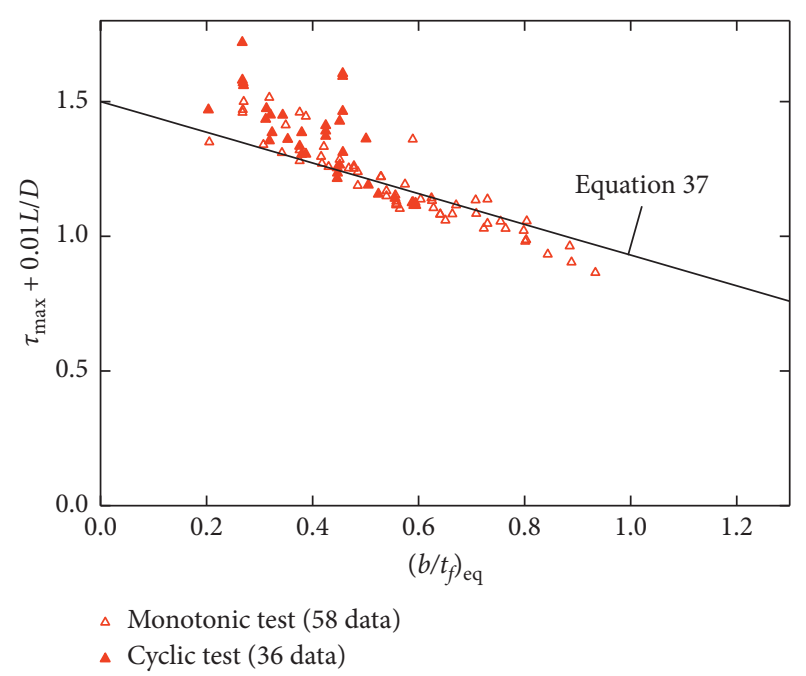

Figure 25: $\tau_{\max }$ versus $\left(\mathrm{b} / t_{f}\right)_{\text {eq }}$ of flange buckling dominant beams.

4.2. Web Buckling Slenderness Ratio and New Design Equation. To evaluate the ultimate strength of a web buckling dominant $\mathrm{H}$-section beam, not only the depththickness ratio but also other parameters such as aspect ratio, bending gradient, and section areas should be considered. To avoid complex calculations, a direct strength method based on the calculation of elastic buckling stress is employed to investigate the relationship between the normalized ultimate strength $\tau_{\max }$ and web buckling slenderness ratio $S_{w}$, in which $S_{w}$ is defined as follows:

$$
S_{w}= \begin{cases}\sqrt{\sigma_{\mathrm{wy}} / \sqrt{3} \tau_{\mathrm{crw}}}, & \left(Q_{p} \geq_{w} Q_{p}\right), \\ \sqrt{M_{p} / M_{\mathrm{crw}}}, & \left(Q_{p}<{ }_{w} Q_{p}\right),\end{cases}
$$

where $\tau_{\text {crw }}$ is the shear buckling stress which is calculated by using equations (13) and (30); $M_{\text {crw }}$ is the bending buckling moment which is calculated by using equations (12), (29), and (39):

$$
M_{\mathrm{crw}}=\sigma_{\mathrm{crw}}\left[B t_{f} d+\frac{t_{w}\left(d-t_{f}\right)^{2}}{4}\right]
$$

By changing the abscissa of Figure 24 into $S_{w}$, Figure 26 can be obtained. By comparison, the data dispersion in Figure 26 is smaller than that in Figure 24 when $\sigma_{\text {crw }} \leq 1.5 \sigma_{\text {crf }}$, and it is reasonable to suppose that the local buckling is dominated by web buckling when $\sigma_{\text {crw }} \leq 1.5 \sigma_{\text {crf }}$. Therefore, the new defined web buckling slenderness ratio $\left(S_{w}\right)$ can be regarded as a major parameter to evaluate the normalized ultimate strength $\left(\tau_{\max }\right)$ of a web buckling dominant $\mathrm{H}$-section beam. However, the test results are lower than the Euler curve equation (40), due to the inelastic buckling:

$$
\tau_{e}=\frac{1}{S_{w}^{2}}
$$

$\tau_{\mathrm{e}}$ is the normalized elastic buckling strength. The following asymptotic equation (41) is attempted to evaluate $\tau_{\max }$, in which $\tau_{\max }$ converges to equation (40) with a large value of $S_{w}$, and $\tau_{\max }$ converges to 1 with a small value of $S_{w}$ :

$$
\frac{1}{\tau_{e}^{2}}+1=\frac{1}{\tau_{\max }^{2}} \text {. }
$$

By substituting equation (40) into equation (41), (41) can be written as

$$
\tau_{\max }=\frac{1}{\sqrt{S_{w}^{4}+1}} .
$$

As shown in Figure 26, equation (42) takes the lower limit in the range of $\sigma_{\mathrm{crw}} \leq 1.5 \sigma_{\mathrm{crf}}$. However, it has been indicated [14] that $\tau_{\max }$ may be larger than 1 for an inelastic buckling $\mathrm{H}$-section beam. To avoid underestimations, equation (43) is attempted:

$$
\tau_{\max }=1.35-S_{w}^{2} .
$$

Figure 27 shows the relationship between $\tau_{\max }$ and $S_{w}$ in the range of $\sigma_{\mathrm{crw}} \leq 1.5 \sigma_{\mathrm{crf}}$ only. By taking the maximum value of equations (42) and (43), (44) is obtained:

$$
\tau_{\max }=\max \left\{1.35-S_{w}^{2}, \quad \frac{1}{\sqrt{S_{w}^{4}+1}}\right\} .
$$

It is shown that proposed equation (44) takes the lower limit of test results, and the upper limit is about its $125 \%$. This means that the deviation caused by initial geometric imperfection and residual stress is lower than $25 \%$ in the tests. The proposed design equation (44) produces good predictions for the test results of the ultimate strengths of the web buckling dominant $\mathrm{H}$-section beams, and the application range is $\sigma_{\text {crw }} \leq 1.5 \sigma_{\text {crf }}$.

Moreover, according to the test data (Figure 27), the normalized ultimate strength $\tau_{\max }$ is not affected by the loading program (including monotonic loading and various kinds of cyclic loading) when the buckling is dominated by web buckling. For both monotonic tests and cyclic tests, the dispersion is small. 


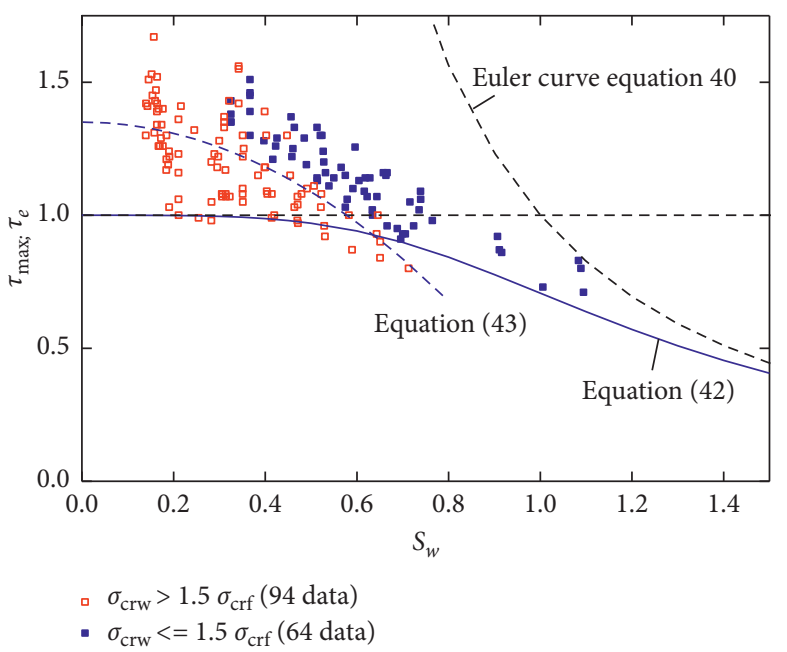

FIgURE 26: $\tau_{\max }$ versus $S_{w}$.

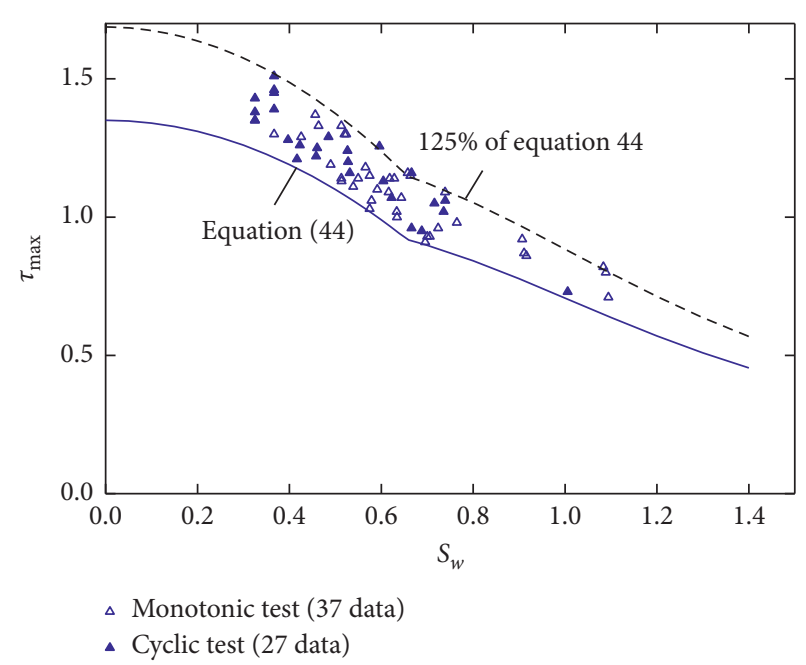

FIgURE 27: $\tau_{\max }$ versus $S_{w}$ of web buckling dominant beams.

The deviation in Figure 27 is about 25\%, meaning that the beams with the same buckling slenderness ratio may have different normalized ultimate strengths with $25 \%$ deviation. In Section 2.1 (Figures 4 and 5), the FEA results have shown that the influence of the geometric imperfections with $D / 800 \sim D / 200$ only causes approximately $5 \%$ deviation, which is far smaller than $25 \%$. In addition, other influences such as residual stresses, material characteristics, and the test methods may also cause deviations to a certain degree. However, these could not be the primary reason for the large deviation. As mentioned previously, the direct strength method is used in this study, which calculates the elastic buckling stress and buckling slenderness ratio to predict the normalized ultimate strength. The beams with the same buckling slenderness ratio do not necessarily mean they have the same normalized ultimate strength. The beams with different configurations may have different buckling behaviors and different normalized ultimate strengths, even though they have the same value of buckling slenderness ratio. Therefore, to improve the evaluation method of the normalized ultimate strength, further parameters and their influences should be studied to reduce the deviation.

\section{Summary and Conclusions}

Theoretic analysis by Ritz's energy method for the H-section beam under combined bending and shear force is presented. The theoretic analysis was verified against the FEA when the buckling of the beam is dominated by web buckling. A parametric study based on the stress separation concept is conducted to simplify the calculation method for buckling coefficients. The design equation based on direct strength method for the normalized ultimate strength of a web buckling dominant $\mathrm{H}$-section beam is proposed. The conclusions are drawn as follows:

(1) Even when the flange is slightly buckled, the buckling mode and buckling stress of an $\mathrm{H}$-section beam web show no obvious difference with a single web with clamped edges, when the local buckling of the beam is dominated by web buckling. The analytic model of a web buckling dominant $\mathrm{H}$-section beam can be simplified by a single web with clamped edges.

(2) Without considering the effect of shear stress, the bending buckling coefficient $k_{\mathrm{bw} 0}$ of web under unequal bending stress is related to the aspect ratio $\lambda_{w}$ and moment gradient $\beta$, and when $\lambda_{w}>1, k_{\mathrm{bw} 0}$ can be approximately expressed by equation (27). The shear buckling coefficients $k_{\text {sw0 }}$ (equation (25)) and bending buckling coefficient $k_{\mathrm{bw} 0}$ (equation (27)) can be regarded as the upper limits of the buckling coefficients $k_{\mathrm{sw}}$ and $k_{\mathrm{bw}}$ of web under combined bending and shear stress, respectively. The interaction curve of the buckling coefficients can be expressed by equation (28), and the approximate equations for calculating the buckling coefficients (equations (29) and (30)) are proposed.

(3) According to a number of tests, it is shown that the normalized ultimate strength $\tau_{\max }$ has a strong correlation with the equivalent width-thickness ratio $\left(b / t_{f}\right)_{\mathrm{eq}}$ in the range of $\sigma_{\mathrm{crw}}>1.5 \sigma_{\mathrm{crf}}$, whereas $\tau_{\max }$ has a strong correlation with the web buckling slenderness ratio $S_{w}$ in the range of $\sigma_{\mathrm{crw}} \leq 1.5 \sigma_{\mathrm{crr}}$. It is reasonable to assume that the local buckling is dominated by the flange buckling when $\sigma_{\text {crw }}>1.5 \sigma_{\text {crf }}$, whereas it is dominated by the web buckling when $\sigma_{\mathrm{crw}} \leq 1.5 \sigma_{\mathrm{crf}}$.

(4) The distribution of the normalized ultimate strength $\tau_{\max }$ obtained by cyclic tests is slightly higher than that obtained by monotonic tests, when the local buckling is dominated by flange buckling. However, $\tau_{\max }$ is not affected by the loading program when the buckling is dominated by web buckling.

(5) A new design equation (equation (44)) to evaluate the ultimate strength of a web buckling dominant $\mathrm{H}$-section beam is proposed, which showed sound agreements with test results. 


\section{Nomenclature}

\begin{tabular}{|c|c|}
\hline$\lambda_{\mathrm{w}}:$ & Aspect ratio of web, $\lambda_{\mathrm{w}}=L / \mathrm{d}$ (Figure 2 ) \\
\hline$\beta:$ & Moment gradient (Figure 2) \\
\hline $\mathrm{d} / t_{\mathrm{w}}:$ & Depth-thickness ratio of web (Figure 2) \\
\hline $\mathrm{b} / t_{f}$ & Width-thickness ratio of flange (Figure 2) \\
\hline $\begin{array}{l}A_{w}=d t w \\
A f=B t f:\end{array}$ & Section area of web and flange (Figure 2) \\
\hline$D_{w}:$ & Flexure rigidity of web (equation (11)) \\
\hline$E:$ & Young's modulus, $E=2.05 \times 105 \mathrm{MPa}$ \\
\hline$v:$ & Poisson ratio $v=0.3$ \\
\hline$M_{\mathrm{b}}$ & Bending moment on the left end (Figure 2) \\
\hline$M_{\mathrm{b}}:$ & Full plastic bending moment (equation (32)) \\
\hline$M_{\text {crw }}:$ & Bending buckling moment (equation (39)) \\
\hline$Q_{s}:$ & Shear load (Figure 2) \\
\hline$Q_{P}:$ & $\begin{array}{l}\text { Shear strength in the full plastic bending } \\
\text { state (equation (33)) }\end{array}$ \\
\hline wQP: & Yield shear strength (equation (34)) \\
\hline$\sigma_{b}:$ & $\begin{array}{l}\text { Maximum value of bending normal stress } \\
\text { (Figure 7) }\end{array}$ \\
\hline$\tau_{s}:$ & Uniformly distributed shear stress (Figure 7) \\
\hline$\alpha:$ & Ratio of $\tau s$ to $\sigma b$ (equation (6)) \\
\hline$\sigma_{\mathrm{crw}}:$ & $\begin{array}{l}\text { Bending buckling stress (equation (12)), } \\
\text { critical value of } \sigma b\end{array}$ \\
\hline$\tau_{\text {crw }}:$ & $\begin{array}{l}\text { Shear buckling stress (equation (13)), critical } \\
\text { value of } \tau s\end{array}$ \\
\hline$k_{\mathrm{bw}}, k_{\mathrm{sw}}:$ & Buckling coefficient due to $\sigma_{\text {crw }}$ and $\tau_{\text {crw }}$ \\
\hline$k_{\mathrm{bw} 0}:$ & $\begin{array}{l}\text { Buckling coefficient due to } \sigma b \text { in the case of } \\
\tau s=0\end{array}$ \\
\hline$k_{\mathrm{sw0} 0}:$ & $\begin{array}{l}\text { Buckling coefficient due to } \tau \mathrm{s} \text { in the case of } \\
\sigma \mathrm{b}=0\end{array}$ \\
\hline$\sigma_{\text {crf }}$ & Buckling stress of flange (equation (35)) \\
\hline$\sigma_{\mathrm{wy}}, \sigma_{\mathrm{fy}}:$ & Yield stress of web and flange \\
\hline$M_{\mathrm{u}}, Q_{\mathrm{u}}:$ & $\begin{array}{l}\text { Ultimate bending strength and shear } \\
\text { strength }\end{array}$ \\
\hline$\tau_{\max }:$ & $\begin{array}{l}\text { Normalized ultimate strength (equation } \\
(31))\end{array}$ \\
\hline$\tau_{\mathrm{e}}:$ & $\begin{array}{l}\text { Normalized elastic buckling strength } \\
\text { (equation (40)) }\end{array}$ \\
\hline$\left(\mathrm{b} / t_{f}\right)_{\mathrm{eq}}:$ & $\begin{array}{l}\text { Equivalent width-thickness ratio (equation } \\
\text { (36)) }\end{array}$ \\
\hline$S_{\mathrm{w}}:$ & $\begin{array}{l}\text { Web buckling slenderness ratio (equation } \\
(38) \text { ). }\end{array}$ \\
\hline
\end{tabular}

\section{Data Availability}

The data used to support the findings of this study are available from the corresponding author upon request.

\section{Conflicts of Interest}

The authors declare that they have no conflicts of interest.

\section{Acknowledgments}

This work was partially supported by the National Natural Science Foundation of Zhejiang Province, China (Grant no. LQ19E080008).

\section{References}

[1] S. P. Timoshenko, "Einge stabilitatsprobleme der Elastizitatstheorie," Zeitschrift für Angewandte Mathematik und Physik, vol. 58, 1910.

[2] R. V. Southwell and S. Skan, "On the stability under shearing force of a flat elastic strip," Proceedings of the Royal Society, vol. A105, 1924.

[3] W. Moheit, "Schubbeulung rechteckiger platten mit eingespannten randern," Thesis, Technische Hochschule Darmstadt, Leipzig, Germany, 1939.

[4] P. P. Bijlaard, "Buckling of plates under nonhomogeneous stress," Journal of the Engineering Mechanics Division, vol. 83, no. EM3, 1957.

[5] D. O. Brush and B. O. Almroth, Buckling of Bars, Plates and Shells, McGraw-Hill, New York, NY, USA, 1975.

[6] S. Yuan and Y. Jin, "Computation of elastic buckling loads of rectangular thin plates using the extended Kantorovich method," Computers \& Structures, vol. 66, no. 6, pp. 861-867, 1998.

[7] J.-H. Kang and A. W. Leissa, "Exact solutions for the buckling of rectangular plates having linearly varying in-plane loading on two opposite simply supported edges," International Journal of Solids and Structures, vol. 42, no. 14, pp. 4220-4238, 2005.

[8] P. Jana and K. Bhaskar, "Stability analysis of simply-supported rectangular plates under non-uniform uniaxial compression using rigorous and approximate plane stress solutions," ThinWalled Structures, vol. 44, no. 5, pp. 507-516, 2006.

[9] K. Ikarashi and T. Suzuki, "An analytical method for elastic buckling strength of clumped plate under unequal bending and shear," Journal of Structural and Construction Engineering (Transactions of AIJ), vol. 68, no. 571, pp. 137-144, 2003.

[10] Y. G. Liu and M. N. Pavlović, "Elastic stability of flat rectangular plates under patch compression," International Journal of Mechanical Sciences, vol. 49, no. 8, pp. 970-982, 2007.

[11] Y. G. Liu and M. N. Pavlović, "A generalized analytical approach to the buckling of simply-supported rectangular plates under arbitrary loads," Engineering Structures, vol. 30, no. 5, pp. 1346-1359, 2008.

[12] T. Suzuki and K. Ikarashi, "Stress separation concept for plate buckling strength under flexural-shear and axial force," Journal of Constructional Steel Research, vol. 70, pp. 317-325, 2002.

[13] A. Kadono, M. Sasaki, K. Okamoto, H. Akiyama, C. Matsui, and K. Inoue, "Experimental study on the effect of yield ratio on the bending strength increasing ratio and the ductility of steel structures' method," Journal of Structural Engineering, AIJ, vol. 40, pp. 673-682, 1994.

[14] Y. Kimura, "Effect of loading hysteretic program on plastic deformation capacity and cumulative plastic deformation capacity for H-shaped beam with local buckling," Journal of Structural and Construction Engineering (Transactions of AIJ), vol. 76, no. 664, pp. 1143-1151, 2011.

[15] Y. Fukuchi and M. Ogura, "Experiment studies on local buckling and hysteretic characteristics of H-shape beams," Journal of Structural and Construction Engineering, vol. 40, no. 228, pp. 65-71, 1975.

[16] B. Kato, H. Akiyama, and Y. Obi, "Deformation characteristics of $\mathrm{H}$-shaped steel members influenced by local buckling," Journal of Structural and Construction Engineering, vol. 42, pp. 49-58, 1977. 
[17] T. Suzuki, T. Ono, and Y. Kanebako, "The local buckling and inelastic deformation capacity of steel beams under shear bending," Journal of Structural and Construction Engineering, vol. 42, no. 260, pp. 91-98, 1977.

[18] T. Fujikawa and K. Fujiwara, "A study on strength and deformation capacity of h-shaped steel beams under cyclic and reversed loadings," in Proceeding of the architectural research meetings in Kinki chapter, pp. 337-340, Kinki, Japan, 1984.

[19] K. Fujiwara and S. Kato, "A study on deformation capacity of h-shaped beams with relative large ratio of web depth to thickness under cyclic loading," in Proceeding of the Architectural Research Meetings in Kinki Chapter, pp. 337-340, Kinki, Japan, 1985.

[20] K. Yoda, K. Imai, Y. Kurobane, and K. Ogawa, "Bending capacity of thin-walled welded H-section beams," Journal of Structural and Construction Engineering, vol. 54, no. 397, pp. 60-72, 1989.

[21] S. Konomi, K. Sera, T. Ninakawa, Y. Yabe, T. Terada, and S. Sakamoto, "Structure behavior of h-shaped beam-end connected to rhs-column - part1: outline of tests and results," in Proceedings of the Summaries of Technical Papers of Annual Meeting, pp. 1541-1542, Hokuriku, Japan, 1992.

[22] T. Makishi, N. Yamamoto, S. Tsutsui et al., "Experimental study on the deformation capacity of welded beam end connection without weld access hole-part 1: test program," in Proceedings of the Summaries of Technical Papers of Annual Meeting, pp. 1257-1258, Kanto, Japan, 1993.

[23] N. Yamamoto, T. Makishi, K. Fujisawa et al., "Experimental study on the deformation capacity of welded beam end connection without weld access hole - Part 2: test results and discussion," in Proceedings of the Summaries of Technical Papers of Annual Meeting, pp. 1259-1260, Kanto, Japan, 1993.

[24] M. Yoshikawa, T. Suzuki, T. Ogawa, K. Kimura, S. Motoyui, and T. Sueoka, "Investigation on estimation of inelastic behavior of $\mathrm{H}$-shaped beam with circular hole - part1: influence of geometrical factors in circular hole," in Proceedings of the Summaries of Technical Papers of Annual Meeting, vol. 2, pp. 1473-1474, Kanto, Japan, 1993.

[25] H. Ito, M. Kimura, H. K. T. Yagi, and O. Ishii, "Experimental study on beam-to-column welding connection without beam scallops," in Proceedings of the Summaries of Technical Papers of Annual Meeting, vol. 2, pp. 1261-1262, Kanto, Japan, 1993.

[26] T. Suzuki, S. Motoyuki, T. Fukasawa, and M. Uchikoshi, "Study on effect of width-thickness ratio on deformation capacity of beams with scallops," Journal of Structural and Construction Engineering (Transactions of AIJ), vol. 61, no. 486, pp. 107-114, 1996.

[27] T. Suzuki, K. Ikarashi, T. Azuma, and K. Satsukawa, "A study on plastic deformation capacity of $590 \mathrm{~N} / \mathrm{mm}^{2}$ high strength steel beams with large depth-thickness ratio," Journal of Structural and Construction Engineering (Transactions of AIJ), vol. 64, no. 522, pp. 113-119, 1999.

[28] T. Suzuki, K. Ikarashi, and Y. Tsuneki, "A study on collapse mode and plastic deformation capacity of $\mathrm{H}$-shaped steel beams under shear bending," Journal of Structural and Construction Engineering (Transactions of AIJ), vol. 66, no. 547, pp. 185-191, 2001.

[29] K. Minami, T. Nakagomi, T. Hujita, M. Murai, and Y. Sasaki, "Experimental study on effect of non-scallops on deformation capacities of beam-to-column welded joints-part1: using H-rolled beam with SM490A class steel," in Proceedings of the Summaries of Technical Papers of Annual Meeting, pp. 631-632, Kanto, Japan, 1996.
[30] K. Ikarashi, T. Otabe, and T. Wang, "Plastic deformation capacity and post-buckling behavior of $\mathrm{H}$-shaped beam with large depth-thickness ratio under cyclic loading," Journal of Structural and Construction Engineering (Transactions of AIJ), vol. 74, no. 646, pp. 2345-2354, 2009.

[31] PRC National Standard, "Code for Design of Steel Structures," GB 50017-2003, China Building Industry Press, Beijing, China, 2003.

[32] M. Shokouhian, Y. Shi, and M. Head, "Interactive buckling failure modes of hybrid steel flexural members," Engineering Structures, vol. 125, pp. 153-166, 2016.

[33] M. S. Deepak and V. M. Shanthi, "Distortional buckling-moment resistance capacity of hybrid double-I-box beams," Journal of Structural Engineering, vol. 144, no. 9, p. $04018132,2018$. 